\title{
A Passenger-to-Driver Matching Model for Commuter Carpooling: Case Study and Sensitivity Analysis
}

\author{
Xiaobing LIU ${ }^{1}$, Helena TITHERIDGE ${ }^{2}$, Xuedong YAN $^{1 *}$, Rui WANG $^{3}$, Weimin TAN ${ }^{4}$, Deqi \\ $\mathrm{CHEN}^{1}$, Jiechao $\mathrm{ZHANG}^{5}$ \\ 1 MOT Key Laboratory of Transport Industry of Big Data Application Technologies for \\ Comprehensive Transport, School of Traffic and Transportation, Beijing Jiaotong University, \\ Beijing 100044, China \\ 2 Centre for Transport Studies, University College London, Gower Street, London, WC1E 6BT, UK \\ 3 School of Civil Engineering, Beijing Jiaotong University, Beijing, 100044, China \\ 4 School of Management, Hefei University of Technology, Hefei 230009, China \\ 5 University of Central Florida, Orlando, FL 32816-2450, USA \\ * Correspondence: xdyan@bjtu.edu.cn
}

\section{Abstract}

For the transport sector, promoting carpooling to private car users could be an effective strategy over reducing vehicle kilometers traveled. Theoretical studies have verified that carpooling is not only beneficial to drivers and passengers but also to the environment. Nevertheless, despite carpooling having a huge potential market in car commuters, it is not widely used in practice worldwide. In this paper, we develop a passenger-to-driver matching model based on the characteristics of a private-car based carpooling service, and propose an estimation method for time-based costs as well as the psychological costs of carpooling trips, taking into account the potential motivations and preferences of potential carpoolers. We test the model using commuting data for the Greater London from the UK Census 2011 and travel-time data from Uber. We investigate the service sensitivity to varying carpooling participant rates and fee-sharing ratios with the aim of improving matching performance at least cost. Finally, to illustrate how our matching model might be used, we test some practical carpooling promotion instruments. We found that higher participant role flexibility in the system can improve matching performance significantly. Encouraging commuters to walk helps form more carpooling trips and further reduces carbon emissions. Different feesharing ratios can influence matching performance, hence determination of optimal pricing should be based on the specific matching model and its cost parameters. Disincentives like parking charges and congestion charges seem to have a greater effect on carpooling choice than incentives like preferential parking and subsidies. The proposed model and associated findings provide valuable insights for designing an effective matching system and incentive scheme for carpooling services in practice.

Key words: carpooling, commuter, matching model, generalized trip cost, sensitivity, promotion instruments

\section{Introduction}

Urban roads worldwide are frequently associated with high levels of pollution and congestion. Private car use accounts for the largest portion of kilometers traveled across all travel modes, making it one of the most important contributors to air pollution 
(Lau et al., 2008). In the United Kingdom 93\% of greenhouse gas emissions from the transport sector are attributed to road transport (DfT, 2017a). Around 25\% of total vehicle miles traveled in the UK are for commuting and $85 \%$ of commuters drive alone to work (DfT, 2017b). It is, therefore, vital that a switch to more sustainable transportation modes (e.g. public transportation, bicycle, carpooling) is achieved, especially for single occupancy car commuters. However, there are still numerous road users who are car-dependent, either through personal choice or from being constrained by public transit circumstances (Mcintosh et al., 2014; Stiglic et al., 2018). Carpooling as a travel means is more flexible than transit and less expensive than traditional taxis, and has been recognized as a potential solution for mitigating the car-dependency problem (Chan and Shaheen, 2012; Bachmann et al., 2018). In recent years, with the growth and acceptance of the sharing economy, the popularity of mobile internet technology, as well as the application of innovative technologies (Dong et al., 2018), internet-based carpooling has emerged in many cities. Internet-based carpooling platforms can effectively match unacquainted drivers and passengers in terms of both time and routes, making scale development of carpooling possible (Furuhata et al., 2013). Nevertheless, even though carpooling services can relieve the most pressing transport problems and has a huge potential market in car commuters (Hong et al., 2017), this travel mode is still not sufficiently used in practice (Delhomme and Gheorghiu, 2016).

From the perspective of transport regulators, the lack of effective incentives designed to make carpooling more attractive to drivers could contribute to the low carpooling usage rate. In order to boost carpooling, a number of measures have been proposed in cities worldwide, including financial incentives attributed to carpooling parking charges (Vanoutrive et al. 2012) or directly allocated to carpooling trips (Liu et al, 2019). Additionally, High Occupancy Vehicle (HOV) lanes are commonly adopted in western industrialized countries. However, both theoretical and empirical studies have shown that the supposed benefits of HOV lanes are often limited (Kwon and Varaiya, 2008; Wang, 2011). In sum, there is still a lack of consensus as to the most effective measures to boost carpooling.

From the perspective of urban travelers, car-dependent people are frequently less concerned about the environment but more sensitive to privacy issues and convenience in a trip (Correia and Viegas, 2011; Delhomme and Gheorghiu, 2016) than others. The advantages of carpooling are generally not strong enough to entice car-dependent travelers to give up the comfort and flexibility of driving alone (Vanoutrive et al. 2012). The personal negative perceptions and attitudes of car users toward carpooling make it difficult for them to share their empty seats with strangers.

In this work, we propose a passenger-to-driver based matching model for car commuters aimed at developing more sustainable and scalable carpooling services, incorporating the characteristics of a private-car based carpooling service and the 
motivations of potential carpoolers. Using actual car commuting trip data for the Greater London, a system sensitivity analysis is conducted and several different policies for promoting carpooling are examined. These results can provide valuable insights on the designs of an effective matching system and incentive scheme for carpooling services in practice.

The remainder of the paper is structured as follows. In Section 2, we present a literature review focusing on the carpooling mode, matching models and other practical issues. In Section 3, we detail the methodology of modeling driver-to-passenger based carpooling service. Our analysis of the Greater London dataset and the results of a series of experiments based on our model are discussed in Section 4. Finally, Section 5 ends this paper with major conclusions, as well as a discussion of future research.

\section{Literature review}

\subsection{Carpooling service modes}

Carpooling is a means of transportation where at least two carpooling participants with similar itineraries, including route and schedule, share a car for at least a part of their journey. According to a classification by Furuhata et al. (2013), traditional carpooling includes: a) informal carpooling, mainly involving acquaintances like family, colleagues, neighbors, and friends. Among strangers, ad hoc ridesharing (e.g., hitchhiking) has also occurred. However, these types of carpooling activities do not scale well due to limited and inefficient communication methods; b) organized carpooling operated by agencies that provide ride-matching opportunities for participants with no prior connections. Due to this, organized carpooling has great potential as a scalable service. As a relatively novel kind of carpooling service frequently organized by transportation network companies (TNCs) such as Uber and Didi, internet-based carpooling services have become popular in many cities over the last decade (Dong et al., 2018). Table 1 lists several current online carpooling services provided by the major platforms and their respective characteristics, based on data from the official websites of the respective TNCs.

Table 1. The characteristics of internet-based carpooling provided by major platforms

\begin{tabular}{|c|c|c|c|c|}
\hline $\begin{array}{c}\text { Payment } \\
\text { level }\end{array}$ & $\begin{array}{l}\text { Operating } \\
\text { attribute }\end{array}$ & $\begin{array}{c}\text { Detour } \\
\text { agent }\end{array}$ & $\begin{array}{l}\text { Main trip } \\
\text { purposes }\end{array}$ & $\begin{array}{c}\text { Major } \\
\text { platforms }\end{array}$ \\
\hline Fee-sharing & Commercial & Drivers and riders & Diverse & $\begin{array}{c}\text { Uberpool, Didi } \\
\text { Express Pool }\end{array}$ \\
\hline Profitable & Private & Drivers and riders & Diverse & Didi Hitch \\
\hline Cost-sharing & Private & Riders & Intercity & Blablacar \\
\hline Cost-sharing & Private & Riders & Commuting & Waze Carpool \\
\hline
\end{tabular}
into two categories: ride-hailing based carpooling and private-car based carpooling. 
Ride-hailing based carpooling (or ride-splitting) enables two or more groups of riders to share the empty seats in a vehicle and split the fare costs, e.g., Uberpool and Didi Express Pool. The ride-hailing driver is a professional driver with a permit license and provides transportation services for various trip purposes in order to make a profit. In general, the routes of two groups of passengers sharing one trip are not exactly identical; thus the carpooling driver will need to detour between the first group of passengers' origins (destinations) and the second group of passengers' origins (destinations).

In contrast, private-car based carpooling involves non-professional drivers sharing their empty seats with one or more groups of passengers in one private car. Payment from riders is generally just sufficient to lower the costs of travelling but is not enough to provide drivers with a profit (Polkowski and Dysarz, 2016). For example, with Waze Carpool for commuters drivers cannot charge riders more than 0.54 US\$ per mile, which is the 2018 reimbursement rate cap set by the US Internal Revenue Service (IRS) for business travel by $\mathrm{car}^{1}$. Therefore, the drivers using these platforms frequently dictate the whole carpooling journey, setting the meeting points and meeting times based on their own itineraries, while passengers need to make additional effort to reach the meeting points by the agreed times. However, related studies tend to either assume the drivers would detour to pick up or drop-off passengers (Agatz et al., 2011; Amey, 2011; Stiglic et al, 2016), or simply discuss similar service modes but do not go on to model them (Furuhata et al., 2013; Stiglic et al., 2015). Note, the private drivers of Didi Hitch do profit from a carpooling fee and tend to detour to pick up or drop off riders.

For ride-hailing based carpooling, some cities, for example in the United States, have imposed a limited set of regulations on these services (e.g. requiring registration as professional drivers and insurance for commercial operation); regulators of several countries (e.g. France, Spain, and Germany) even have taken action against this type of carpooling service (Cetin and Deakin, 2017). Generally, there seems to be more tolerance of private-car based carpooling than ride-hailing based carpooling services for authorities in practice. If drivers do not meet the criteria of business activity and do not charge passengers above the costs of a ride, then a carpooling service would not need a special permit license to operate and would not challenge current ordinances and laws that would come with the ride-haling services (Chan and Shaheen, 2012; Polkowski and Dysarz, 2016). An additional benefit of private-car based carpooling stems from the fact the participants involved are likely to travel by private car whether there are carpooling services or not, and hence private-car based carpooling has greater potential to reduce vehicle-kilometers traveled. Private-car based carpooling also has greater potential in both numbers of candidate vehicle numbers and numbers of

${ }^{1}$ https://www.irs.gov/tax-professionals/standard-mileage-rates 
available empty seats per vehicle than ride-hailing services. Therefore, in this paper we focus on exploring a matching model for a private-car based carpooling service.

\subsection{Matching objectives and generalized trip costs}

An internet-based carpooling platform with the function of centralized control can help match carpooling drivers with riders for the purpose of profit by commissions or advertisement (Agatz et al, 2012). For example, a carpooling provider may charge a service fee per successful carpooling trip, either as a percentage of the trip cost or as a fixed fee. For the overall matching objective in such a carpooling system, most scholars consider one (or a combination) of the following three objectives for the system optimum: 1) to minimize system-wide vehicle kilometers (e.g., Agatz et al., 2011; Wang et al., 2018), 2) to minimize system-wide travel time or cost (e.g., Winter and Nittel, 2006; Long et al, 2019 ), and 3) to maximize the number of participants (e.g., Stiglic et al., 2016; Masoud and Jayakrishnan, 2017). According to previous works, there were two main motivations for car users to shift to carpooling services: monetary cost savings and psychological benefits from protecting the environment (Canning et al., 2010; Delhomme and Gheorghiu, 2016). Hence, it is necessary to introduce cost savings into the system matching objective and to consider the environmental benefits from co-travelling in any trip cost estimations.

Compared with single occupancy car trips, carpooling trips frequently generate several additional costs linked to sharing trips with strangers (Vanoutrive et al. 2012; Hong, et al., 2017). More specifically, some scholars have indicated that one of the key reasons impeding car-users from becoming carpoolers is the psychological losses due to ride-sharing including a perceived loss of privacy and a loss of feeling in control of the journey (Correia and Viegas; 2011; Delhomme and Gheorghiu, 2016). Others have identified distrust relating to personal security and comfort as some of the most influential obstacles for carpooling, especially when the carpoolers are strangers (Chaube et al., 2010; Wang et al., 2017). When facing traffic mode shift, singleoccupancy-car commuters are also concerned about travel time duration and reliability (Long et al, 2018), additional physical effort expenditure (e.g., walking or biking from their origins to carpooling pick-up points), and personal space limitation (Gardner and Abraham, 2007); all of which will be influenced by ridesharing. Besides driving cost and in-vehicle time (IVT) cost, we therefore also need to consider time deviation costs, physical effort costs and psychological costs in carpooling match modeling.

In generalized trip cost estimation, it is common practice to express valuations of attributes in equivalent monetary units of IVT (Shires and De Jong, 2009; Abrantes and Wardman, 2011; Wardman et al., 2016). For example, Abrantes and Wardman (2011) conducted meta-analysis on 226 related studies and obtained the monetary values of IVT for travel demand modelling in the UK. They used the derived IVT multipliers to express valuations of walk, wait and early or late time, etc., based on the characteristics 
of travelers and trips. Such multipliers are not only transferable across different contexts, but also readily lend themselves to interpretation and assessment.

Regarding psychological costs like trust, privacy and control in carpooling, many scholars argue that they are related to social connectivity levels (Wang et al., 2017; Amirkiaee and Evangelopoulos, 2018). People are significantly less willing to share a ride with strangers than with direct or indirect friends. A higher percentage of direct friends in matches potentially leads to lower psychological costs (Wang et al., 2017). In practice, Chaube et al. (2010) reported that $98 \%$ of the population of Virginia Tech university community would accept a ride from a friend, $69 \%$ accept from an indirect friend, and only $7 \%$ from a stranger; this reflects the impact of social connectivity levels on carpooling matches. However, there is still the lack of quantitative methods to formulate the psychological costs that carpoolers need to pay in carpooling modelling.

\subsection{Key issues in carpooling service operation}

Due to considerable constraints in feasible carpooling trips, successfully matching candidate riders and drivers frequently requires a sufficiently large number of participants (Kamar and Horvitz, 2009). Buliung et al. (2010) stressed the importance of the pool-size effect, finding that a larger pool of employees from the same work environment increases the number of potential carpool partners. Stiglic et al. (2016) explored the impact of participant flexibility and participant density on the matching performance of dynamic ridesharing and found that a small increase in passenger flexibility can significantly increase the expected matching rate, especially when the number of trip announcements in the carpooling system is small. Consequently, it will be interesting to examine the impact of participant flexibilities on the private-car based carpooling system proposed in this paper with differing numbers of carpooling candidates.

Considering that monetary cost savings is one of the main motivations for people's sharing behavior, it is also important to examine the impact of different carpooling fee levels on the carpooling system. However, only a few scarce works on carpooling system design deal with this issue. Like most other studies, Geisberger et al. (2009) suggested dividing the cost of the shared part of the trip evenly between the carpooling participants. Agatz et al. (2011) proposed a way to allocate the costs of the joint trip that is proportional to the distances of the separate trips. Matching agencies frequently implement rule-based pricing using a cost calculation formula specified by the matching agency, where the fee is a function of distance travelled (Furuhata et al. 2013). Nevertheless, all these studies and applications neglect the additional costs of carpooling trips mentioned in section 2.2 when designing pricing rules and examining the impact of varying fee structures on matching performance.

To fill these gaps in the development of a matching model for private-car based carpooling, we propose a passenger-to-driver carpooling model for car commuters, 
which is applied to the Greater London using actual car commuting data. The main contributions of this paper can be summarized as follows:

First, on the basis of a cost-sharing principle, we propose a passenger-to-driver matching model for car commuters which considers the time-based costs, psychological costs and the environmental benefits of carpooling. We formulate this matching model as a mixed-integer linear programming problem and obtain the optimal solutions.

Second, we estimate the time-based costs of commuting trips and formulate the psychological loss costs by the social connectivity between carpoolers as well as quantify the environmental benefits by the carbon emission reduction in this carpooling model. We determine the coefficients of various costs in the form of IVT multipliers.

Third, we apply the matching model to the Greater London using data on actual commuting trips. The dataset is established by linking small-zone commuting population data with road network performance data. Additionally, we quantify the effect of a selection of carpooling incentive policies for the Greater London.

Fourth, we investigate the sensitivity of the matching model to varying carpooling participant rates and diverse fee-sharing rules to improve matching performance with least cost.

\section{Methodology}

\subsection{Passenger-to-driver matching mechanism of private-car based carpooling}

In this paper, we seek to explore the maximally achievable goals of our proposed matching model while ensuring these solutions provide useful insights for practice. Thus, we introduce a potential carpooling system which provides information to our matching model and conduct our analysis with the perspective of service provider in mind. Note we do not intent to design a matching system to support a carpooling services platform but instead to use the outline of the carpooling system as a tool to estimate the carpooling matching upper-bound.

In general, a complete private-car based carpooling trip is assumed to form and execute as follows. First, both carpooling drivers and riders launch their itineraries in the carpooling platform in advance. Second, the carpooling system matches feasible trips using a set objective and sends the matching information to carpoolers. Third, according to the meeting time and meeting point recommended by the platform (or negotiated by the participants), the matched driver and rider pairs arrive at their pickup point at their agreed time and travel onwards together. Having arrived at their dropoff point, the riders pay a carpooling fee based on the platform's pricing rule and then the carpoolers continue their respective journeys on to their final destinations.

In this paper, we assume that a carpooling driver only takes a single rider, since the computational complexity for computing all possible routes of one-driver-multiple- 
passengers carpooling trips increases rapidly; this frequently necessitates the deployment of heuristic algorithms. However, this decision may not significantly impact the results. The travel-time flexibility of peak time commuters is often limited; picking up multiple riders may substantially increase travel delays and the inconvenience of carpooling, particularly where commuter densities are low.

As discussed in Section 2.1, it is frequently the drivers who dictate the characteristics of the carpooling journey rather than the riders. Hence, we assume that a driver would travel in accordance with their original route and schedule with no additional detours or delays due to picking up or dropping off a passenger. The meeting point and associated meeting time are determined based on the driver's itinerary. Similarly, for the drop-off point. In our model, the meeting point, meeting time, and drop-off point are assumed to be set by the service platform to minimize the additional effort each passenger undergoes. However, in reality, they could be chosen by the driver and passenger through negotiation or set by the system using more sophisticated criteria. We assumed a carpooling passenger would walk to the nearest pick-up point located along their driver's route, arriving by the agreed time. Likewise, the passenger would walk to their workplace from the nearest drop-off point located along their driver's route. More precisely, the proposed carpooling variation is driver-controlled and a rider frequently needs to pay additional walking effort to reach the meeting point and an additional schedule deviation cost to match the meeting time; this matching mechanism is referred to as the passenger-to-driver matching pattern from herein.

The following notations are adopted throughout this paper:

\begin{tabular}{|c|c|}
\hline$C_{A}, C_{S}$ & Generalized cost of the driving-alone trip $A$ and the ride-sharing trip $S$ \\
\hline$\Delta C^{P}, \Delta C^{D}$ & Generalized trip cost saving of the passengers and drivers in carpooling trip \\
\hline$C S_{D P}$ & Generalized cost saving of the carpooling trip $(D, P)$ \\
\hline$H, W$ & Housing places and workplaces based on communities \\
\hline$N$ & $\begin{array}{l}\text { The market access threshold: the lowest number of car commuting trips for a certain } \\
\text { area to be introduced into the local carpooling market. }\end{array}$ \\
\hline$P F, C b, P C, C F$ & Parking fee; carbon emission cost; psychological cost; carpooling service fee. \\
\hline$T_{t}, T_{w}, T_{d}$ & Travel time in driving-alone trip; walking time; deviation time from the schedule \\
\hline$U_{w}, U_{s}$ & Upper threshold of walking time and upper threshold of time-based search scope \\
\hline$x_{D P}$ & A $0-1$ decision variable; if carpooling trip $(D, P)$ formed, $x_{D P}=1$; otherwise, $x_{D P}=0$ \\
\hline$\chi, \lambda, \alpha, \gamma, \delta, \varepsilon$ & $\begin{array}{l}\text { Unit cost factor of variable driving cost, in-vehicle travel time, psychological penalty, } \\
\text { walking time, schedule deviation penalty at the residence and at the workplace }\end{array}$ \\
\hline$\omega$ & $\begin{array}{l}\text { Shared trip stage ratio of shared driving time (with rider) to the total travel time of the } \\
\text { driver in a carpooling trip }\end{array}$ \\
\hline$\Omega$ & The set of feasible carpooling trips \\
\hline$\theta(\partial)$ & $\begin{array}{l}\text { The angle between the carpooling route vector and the route vector from driver's } \\
\text { housing (workplace) to rider's housing (workplace) }\end{array}$ \\
\hline
\end{tabular}


We assume that all participants in the carpooling system have private cars and would complete their trips by driving alone if without the carpooling service. In general, the generalized cost of a single occupancy car trip consists of three components: 1) the driving cost including fixed driving costs and variable driving costs, 2) the in-vehicle travel time cost, and 3) the external environmental costs (for our purposes defined here as carbon emissions). These three components of a carpooling driver's or passenger's respective trips in case of driving-alone can be formulated in Eq. 1, where the subscript 'A' stands for a driving-alone trip, $P F$ is the parking fee as a fixed driving cost, $\chi_{A}$ is the variable driving cost of unit time including fuel costs, wear and tear costs, insurance, depreciation costs and other kilometer-based costs. $\lambda$ is the unit cost of in-vehicle travel time, $T_{t}^{D}$ and $T_{t}^{P}$ are the in-vehicle travel time of the driver and passenger respectively, $C b_{A}^{D}$ and $C b_{A}^{P}$ are carbon emissions costs of a driver's and passenger's respective trips.

$$
C_{A}^{D}=\left(P F+\chi_{A} T_{t}^{D}\right)+\lambda T_{t}^{D}+C b_{A}^{D} \quad C_{A}^{P}=\left(P F+\chi_{A} T_{t}^{P}\right)+\lambda T_{t}^{P}+C b_{A}^{P}
$$

Besides the above costs involved in a driving-alone trip, we also need to consider the time-deviation cost, the physical effort cost and the psychologist loss cost in a carpooling trip. In our proposed carpooling matching mode, the components of generalized cost of a carpooling trip are different between a driver and a rider. For a driver, there are four main cost components as shown in Eq. 2: 1) the trip driving cost, 2) the in-vehicle travel time cost, 3) the carbon emissions cost, and 4) the sharing psychological penalty cost incorporating distrust of strangers and the privacy loss associated with sharing personal space with passengers. Because the driver does not deviate from their normal route in this model, the driving cost to the carpooling driver is the difference between the driving-alone trip driving cost and the carpooling fee received from their passenger. The subscript ' $S$ ' indicates a ride-sharing trip. The carpooling fee $C F$ includes half of the driving cost of the carpooled trip including the parking fee $P F$ and a variable driving cost $\chi_{s} T_{t}^{D}$. Note that the unit variable driving cost of a carpooling trip $\chi_{S}$ only covers the fuel cost, but does consider the impact of additional passenger's weight on fuel consumption (Jacobson and King, 2009), where $\bar{\chi}$ is the unit fuel cost of driving-alone, $\beta_{1}$ is the unit fuel-used per additional passenger and $k$ is the number of passengers, in this paper $k=1 . C b_{s}$ is the carbon emissions cost of a carpooling trip and we assume the driver and rider share this cost equally. $P C^{D}$ indicates a driver's psychological penalty cost and $\alpha$ is the unit cost factor of psychological penalty.

$C_{S}^{D}=\left(P F+\chi_{A} T_{t}^{D}-C F\right)+\lambda T_{t}^{D}+\frac{1}{k+1} C b_{S}+\alpha P C^{D} ; C F=\frac{1}{k+1}\left(P F+\chi_{S} T_{t}^{D}\right) ; \chi_{S}=\bar{\chi}+\beta_{1} k$

Passengers in a carpooling trip frequently need to adjust their itineraries to match the driver's journey, therefore the total generalized cost consists of six components formulated in Eq. 3: 1) the trip driving cost, i.e. carpooling fee, 2) the in-vehicle travel time cost, 3) the carbon emission cost, 4) the sharing psychological penalty cost 
covering distrust issues privacy loss from sharing personal space and perceived loss of control of the journey, 5) the detour effort penalty cost due to the additional walking, and 6) the schedule deviation penalty including the requirement to leave home and/or reach the destination early or late and. $P C^{P}$ is a passenger's psychological penalty cost, $T_{w}^{H}, T_{w}^{W}$ and $T_{d}^{H}, T_{d}^{W}$ are the walking time and schedule deviation time at the residence and workplace, respectively. The shared trip stage factor $\omega$ is the ratio of shared driving time (with rider) to the total travel time of the driver. $\gamma, \delta$ and $\varepsilon$ are the unit cost factors of walking time, schedule deviation at the residence and schedule deviation at the workplace, respectively.

$C_{S}^{P}=C F+\lambda \cdot \omega T_{t}^{D}+\frac{1}{k+1} C b_{S}+\alpha P C^{P}+\gamma\left(T_{w}^{H}+T_{w}^{W}\right)+\left(\delta T_{d}^{H}+\varepsilon T_{d}^{W}\right)$

With regards to the sharing psychological penalty cost, when a driver and a rider carpooling pair are strangers (i.e. with the lowest level of social connectivity) then the more time spent sharing with each other, the higher the psychological cost to each of them. Conversely, if the driver is acquainted with the passenger, which is possible considering the matched pair are likely to live and work in the same or neighboring communities, the effect of shared time on personal psychological loss will be lower. Therefore, we assume that the psychological penalty cost takes the value of shared trip time divided by a social connectivity level, where the social connectivity level between acquaintances has higher order of magnitude than when between strangers. This is illustrated by Eq. 4, where SCL is the social connectivity level. Note that carpooling passengers have additional control loss compared to drivers, so we introduce an amplification factor $\kappa$ into the estimation of the passenger's psychological cost.

$P C^{D}=\frac{\omega T_{t}^{D}}{S C L} ; P C^{P}=\kappa \cdot \frac{\omega T_{t}^{D}}{S C L}$

According to the Communicate Bond Belong (CBB) theory (Hall \& Davis, 2017), time spent with a person can be conceived as an opportunity cost for developing or continuing relationships. Many scholars also recognized the positive correlation between meeting time and friendship closeness (Roberts \& Dunbar, 2011; Miritello et al., 2013; Hall, 2019). For example, Hall (2019) demonstrated that friendship status is a function of the amount of time spent together and the type of activity, while the amount of time required to shift friendship status is related to individual acceptance ability and is influenced by age, social status and gender, etc. Only considering the shared time during carpooling trips, we use the number of prior times a pair has been matched to represent the time spent together. Hence, we sum the number of times a pair of carpoolers has been matched together with their initial acquaintance level and take an individual friendship acceptance ability factor as the exponent to quantify the social connectivity level $S C L$ in carpooling activities, as shown in Eq. 5. $A L^{\prime}$ represents the initial acquaintance level, which increases by order of magnitude from unacquainted 
carpoolers to well acquainted, familiar carpoolers (e.g., from 0 to 10). $M T$ is the number of times a pair has been matched through the carpooling service. We set an upper threshold $U_{A L}$ for acquaintance level $A L$, as we assume that once a close friendship has developed additional time spend together will not increase the level of acquaintance further, hence the acquaintance level $A L=\min \left\{A L^{\prime}+M T, U_{A L}\right\}$. The exponent $\rho$ is the friendship acceptance ability, set as a positive number less than 1 ; this ensures the social connectivity level does not increase sharply with increasing time spent as a matched pair, especially for carpoolers who initially start as strangers. The higher the value of the exponent $\rho$, the higher the friendship acceptance ability is and the faster the social connectivity level will grow. In practice, internet-based carpooling services could use a similar approach to find matched pairs. All of the parameters for psychological penalty estimation can be calibrated according to users' initial individual preferences and data records. For example, a rider could identify their initial acquaintance level with the potential driver and this acquaintance level will grow as the rider and driver successfully share carpooled trips. Users might also be able to set their individual level of friendship acceptance ability to reflect their willingness to make connection with others they share journeys with. This would have the added benefit of making it easier for the carpooler to find matched trips.

$S C L=A L^{\rho} ; A L=\min \left\{A L^{\prime}+M T, U_{A L}\right\} ; 0<\rho<1$

Based on equations 1 to 5, we can calculate the trip cost saving of a driver as Eq. 6 and the trip cost saving of a passenger as Eq. $7 . C b R_{i j}$ is the total carbon emissions reduction of matched trips $i$ and $j$ (Eq. 10), and is divided between the carpooling driver and passenger, namely through $\triangle C b^{D}$ and $\triangle C b^{P}$ in Eq. 8 and 9 respectively. As discussed earlier, the total carbon emissions reduction here represents the environmental benefit obtained from co-travelling by carpooling. Note that $\beta_{2}$ is the unit carbon emissions per additional $k$ passengers $(k=1)$ and $\xi$ is the carbon reduction cost conversion factor to time units. Finally, the total generalized trip cost saving of both participants is illustrated in Eq. 10, where $C S_{D P}$ represents the collective cost saving from two individuals by matching driver's trip and passenger's trip, including the driving cost saving, in-vehicle time saving, total carbon emissions reduction benefit, sharing psychological penalty, walking effort penalty and schedule deviation penalty.

$$
\Delta C^{D}=C_{A}^{D}-C_{S}^{D}=C F+\Delta C b^{D}-\alpha P C^{D}
$$

$\Delta C^{P}=\left(P F+\chi_{A} T_{t}^{P}-C F\right)+\lambda\left(T_{t}^{P}-\omega T_{t}^{D}\right)+\Delta C b^{P}-\alpha P C^{P}-\gamma\left(T_{w}^{H}+T_{w}^{W}\right)-\left(\delta T_{d}^{H}+\varepsilon T_{d}^{W}\right)$

$$
\Delta C b^{D}=C b_{A}^{D}-\frac{1}{k+1} C b_{S}=\xi T_{t}^{D}-\frac{\xi}{k+1}\left[(1-\omega) T_{t}^{D}+\beta_{2} k \cdot \omega T_{t}^{D}\right]
$$


$\Delta C b^{P}=C b_{A}^{P}-\frac{1}{k+1} C b_{S}=\xi T_{t}^{P}-\frac{\xi}{k+1}\left[(1-\omega) T_{t}^{D}+\beta_{2} k \cdot \omega T_{t}^{D}\right]$

$C S_{D P}=\underbrace{\left(P F+\chi_{A} T_{t}^{P}\right)}_{\text {Driving cost saving }}+\underbrace{\lambda\left(T_{t}^{P}-\omega T_{t}^{D}\right)}_{\text {In-vehicle time saving }}+\underbrace{\left(\Delta C b^{D}+\Delta C b^{P}\right)}_{\text {Carbon reduction }}-\underbrace{\alpha\left(P C^{D}+P C^{P}\right)}_{\text {Psychological penalty }}-\underbrace{\gamma\left(T_{w}^{H}+T_{w}^{W}\right)}_{\text {Walking penalty }}-\underbrace{\left(\delta T_{d}^{H}+\varepsilon T_{d}^{W}\right)}_{\text {Scheduel deviation penalty }}$

400

401

402

403

404

405

406

407

408

409

410

411

412

413

414

415

416

417

418

419

420

421

422

423

424

425

426

427

428

429

430

431

432

The trip driving cost and all unit variable cost coefficients can be estimated using real data or by referring to previous academic literature. For the schedule deviation cost of a carpooling passenger, it is assumed that the rider would depart earlier or later to match the meeting time proposed by the driver. Therefore, departing deviation time is the difference between the time of a driver arriving at the pick-up point and the time a passenger arrives at it based on the passenger's original (pre-carpooling) start time (shown in Eq. 11), where $\tau^{D}$ and $\tau^{P}$ are the start time of a driver and a passenger when driving alone, respectively. Arriving schedule deviation is the difference between the time a passenger arrives at their workplace by carpooling and the time a passenger would arrive if they drove alone to the workplace (shown in Eq. 12). $\quad \eta$ is the ratio between the drive time from a driver's residence to the pick-up point and the driver's total driving time. Considering the distinction between the unit cost of a late penalty and an early penalty at the residence and workplace (Abrantes and Wardman, 2011; Long, et al., 2018), we take $\delta^{+}\left(\varepsilon^{+}\right)$and $\delta^{-}\left(\varepsilon^{-}\right)$as the penalty factors for leaving the residence (arriving at the workplace) later or earlier than planned respectively. Note that all these factors can be expressed in equivalent units of in-vehicle time (IVT), namely by IVT multipliers. However, the specific walking time needs to be further explored based on our dataset.

$\delta T_{d}^{H}=\delta^{+} \max \left\{\left(\tau^{D}+\eta T_{t}^{D}\right)-\left(\tau^{P}+T_{w}^{H}\right), 0\right\}+\delta^{-} \max \left\{\left(\tau^{P}+T_{w}^{H}\right)-\left(\tau^{D}+\eta T_{t}^{D}\right), 0\right\}$

$\varepsilon T_{d}^{W}=\varepsilon^{+} \max \left\{\left(\tau^{D}+T_{t}^{D}+T_{w}^{W}\right)-\left(\tau^{P}+T_{t}^{P}\right), 0\right\}+\varepsilon^{-} \max \left\{\left(\tau^{P}+T_{t}^{P}\right)-\left(\tau^{D}+T_{t}^{D}+T_{w}^{W}\right), 0\right\}$

\subsection{Identification of feasible carpooling trips}

Before establishing a carpooling matching model, it is necessary to define the set of shareable carpooling trips. On a carpooling service platform, each user can claim one of three roles: (1) a driver, (2) a passenger, and (3) either a driver or a passenger. Let $\Gamma, \Gamma_{D}, \Gamma_{P}, \Gamma_{D / P}$ respectively be the set of carpooling users (where $\left.\Gamma=\Gamma_{D} \cup \Gamma_{P} \cup \Gamma_{D / P}\right)$, the set of participants who select to be a driver, the set of participants who select to be a passenger, and the set of participants who are willing to be either a driver or a rider.

A feasible carpooling trip is one where, first, both the driver and rider can save on the trip cost as a result of the shift to carpooling. Second, additional time the passenger spends travelling to meet the driver and travelling from the drop-off point to their workplace should be reasonable. We assume that all car commuters registered on the platform who meet these two conditions are willing to share their trips. 
To ensure the similarity of carpoolers' routes, we only search and match trips from and to the same or adjacent communities, shown as Fig. 1, where community is analogous to a traffic analysis zone, postcode or census tract (this is for convenience as data on commuting is often available for these geographical units, as for our case study). Measuring the initial search scope of feasible trips by travel time, carpooling participants who live and work in neighboring communities that are reachable within a reasonable walking time are considered candidate carpooling partners.

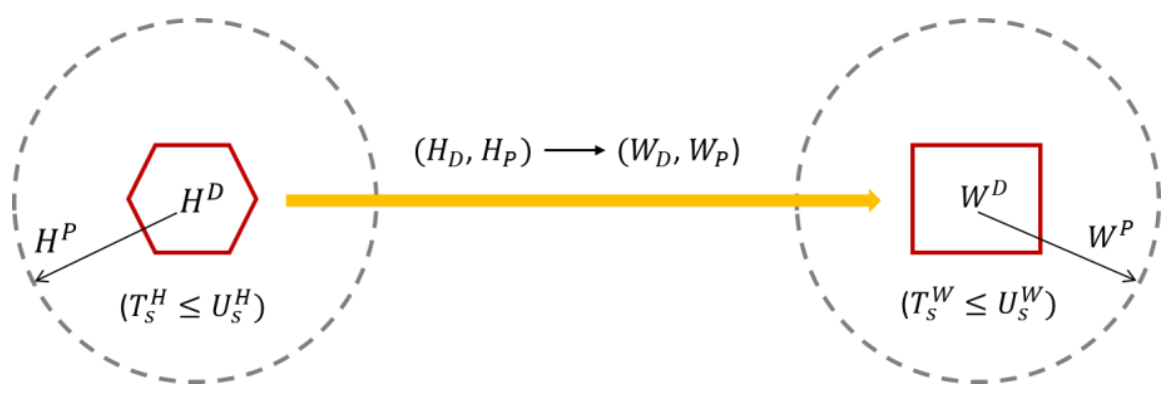

Fig.1 The matching search scope measured by travel time $T_{s}^{H}\left(T_{s}^{W}\right)$ from drivers' housing community (working community $W^{D}$ ) to passengers' housing community $H^{P}$ (working community $\left.W^{P}\right)$ is less than time threshold $U_{s}^{H}\left(U_{s}^{W}\right)$.

Let traveler $D$ be a driver and traveler $P$ be a passenger, hence the set of feasible carpooling trips can be defined by Eq. 13, where $U_{w}$ is the upper threshold of walking time and $U_{s}$ is the upper threshold of the search scope for feasible trips within adjacent communities. Search scope $T_{s}$ is measured by the reasonable walking time between two communities.

$$
\Omega=\left\{(D, P) \mid \Delta C^{D}>0, \Delta C^{P}>0, T_{w} \leq U_{w}, T_{s} \leq U_{s} \text { and } P \neq D, \forall D \in \Gamma_{D} \cup \Gamma_{D / P}, \forall P \in \Gamma_{P} \cup \Gamma_{D / P}\right\}
$$

Based on the set of feasible carpooling trips, we can define the set of candidate drivers $\Theta_{D}$ and the set of candidate passengers $\Xi_{P}$ in Eq.14 and Eq.15, respectively. Combining the shareable carpooling trips set and carpooling candidate set, we can define the set of all candidate passengers for each carpooling driver $\Psi_{D}$ and the set of all candidate drivers for each carpooling passenger $\Phi_{P}$ in Eq.16 and Eq.17, respectively.

$\Theta_{D}=\left\{D \mid \exists P \in \Gamma_{P} \cup \Gamma_{P / D}\right.$ such that $\left.(D, P) \in \Omega\right\}$

$\Xi_{P}=\left\{P \mid \exists D \in \Gamma_{D} \cup \Gamma_{P / D}\right.$ such that $\left.(D, P) \in \Omega\right\}$

$$
\Psi_{D}=\{P \mid(D, P) \in \Omega\}, \forall D \in \Theta_{D}
$$

$$
\Phi_{P}=\{D \mid(D, P) \in \Omega\}, \forall P \in \Xi_{P}
$$

\subsection{Passenger-to-driver matching model}

In this carpooling system, we suppose an internet based carpooling platform charges a service fee per successful carpooling trip as a percentage of the trip cost savings. The carpooling provider would match feasible trip pairs to pursue the 'upper 
$\varpi_{M}=\frac{\sum_{(D, P) \in \Omega} 2 x_{D P}^{*}}{|\Gamma|} \times 100 \%$ 495

$\operatorname{Max} \sum_{(D, P) \in \Omega} C S_{D P} x_{D P}$ variables. cost savings.

bound' of operating profits. Moreover, cost savings are regarded as one of the primary motivations for single occupancy car users shifting to carpooling services. Therefore, here we take the maximal total cost savings of carpoolers as the system objective. As a result, the specific trip cost savings of individual carpoolers are not in an equilibrium situation; some carpoolers can save more from carpooling, while some may scarcely save anything. Based on the set of shareable ride-sharing trips, the set of candidate drivers, and the set of candidate passengers, the carpooling matching model is a weight optimization problem to explore maximally achievable goals. This can be formulated as a mixed-integer linear programming (MILP) problem. The objective function and constraint conditions are listed as follows:

st. $\left\{\begin{array}{l}\sum_{P \in \Psi_{D}} x_{D P} \leq 1, \forall D \in \Theta_{D}, \\ \sum_{D \in \Phi_{P}} x_{D P} \leq 1, \forall P \in \Xi_{P} \text { and } \\ x_{D P}=\{0,1\}, \forall(D, P) \in \Omega\end{array}\right.$

The Objective (18) is to maximize a weighted generalized trip cost saving. Constraint (19) guarantees that each driver is matched with just one passenger and Constraint (20) guarantees that each passenger is matched with just one driver. Constraint (21) is the definitional constraint for the carpooling trip matching decision

To evaluate the matching performance under various scenarios, we define four ratio indexes relating to carpooling matched number, cost savings, carbon emissions reduction, and the matching equity of carpoolers. More specifically, index (22) $\varpi_{M}$ is the carpooling match rate, index (23) $\varpi_{C S}$ is the generalized cost saving rate, and index (24) $\varpi_{C b R}$ is the carbon emission reduction rate, where the asterisk indicates matched carpooling trips and their associated attributes such as trip cost and carbon emissions. $i$ is a participant in a carpooling platform. Index $\varpi_{E}$ (25) is the cost-based carpooling equity factor, defined as the ratio of riders' cost savings to total cost savings in a successful carpooling trip (where passenger number $k=1$ ) to show the fairness between drivers and riders in this matching model from the perspective of individual

$\varpi_{C S}=\frac{\sum_{(D, P) \in \Omega} C S_{D P} x_{D P}^{*}}{\sum_{i \in \Gamma} C_{A}^{i}} \times 100 \%$ 
$\varpi_{C b R}=\frac{\sum_{(D, P) \in \Omega} C b R_{D P} x_{D P}^{*}}{\sum_{i \in \Gamma} C b_{A}^{i}} \times 100 \%$

$\varpi_{E}=\frac{\Delta C^{P}}{C S_{D P}}, \forall(D, P) \in \Omega$

\subsection{Application to the Case of London}

To demonstrate the properties of the proposed model, we solve the MILP problem and analyze the matching performances using empirical parameters and actual car commuting trips data for the Greater London.

London, also referred to as the Greater London, is the largest city in the United Kingdom, with the largest municipal population in the European Union ${ }^{2}$. According to the London Travel Demand Survey (LTDS) (TfL, 2018), the number of trips made in London in 2017 averaged 26.8 million per day. Although London has highly developed public transport network, the mode share of private motorized transport remains above one third of total trips. In Outer London, where public transport coverage is less comprehensive, about half of trips are made by private transport modes (TfL, 2018). The London Mayor's aim for 2041 is for 80 percent of trips in London to be made by active, efficient and sustainable modes and, more ambitiously, to be a zero-carbon city by 2050, despite a growing population (GLA, 2018). For the transport sector, promoting carpooling for private car users to reduce the vehicle kilometers traveled and fuel used, is likely to be part of an effective package of solutions.

\subsubsection{Dataset}

Focusing on commuter carpooling, we extract data on the commuting behavior of full-time workers by location of usual residence and workplaces in London from the United Kingdom Census, $2011^{3}$. In this origin-destination dataset, commuters are divided by usual method of travel to work including private car, public transport, walking, and so on; we take the private car users as carpooling candidates. The population data has an output area (OA) resolution, which is the lowest level of geography produced across all Census topics. To improve the reporting of small area statistics, the reported location of usual residence and workplaces correspond to a middle layer super output area (MSOA). Each MSOA possesses a population of 5000 to 15000 , which is about 25 times the OA population. London is composed of 982 MSOAs with an average area of $1.62 \mathrm{~km}^{2}$. A moderately sized set of representative

2 http://worldpopulationreview.com/regions/european-union-population/

3 https://data.gov.uk/dataset/150b43db-10ce-465d-9961-29e679350a9d/2011-census 
MSOAs, whose areas are around this average value, are shown in Fig. 2 with MSOA codes and a length scale.

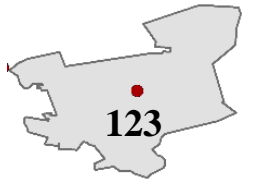

Fig. 2 Four representative MSOAs with are
are the area-based centroids of these zones.

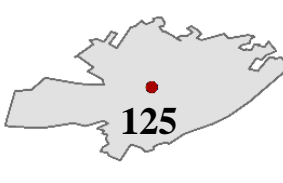

07501,500
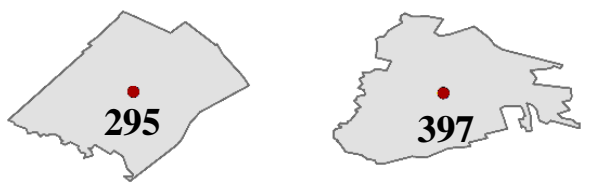

- Centroid

To obtain the carpooling matching costs, we need information about the local road network service performance for private cars. Uber Movement ${ }^{4}$, an open data platform provided by Uber Company, provides zone-to-zone travel time data across the city, and for London at MSOA level. Specifically, this dataset records trip information between origin MSOAs and destination MSOAs including average travel times and standard deviation of travel times from 2016 to the present by start-time. The average travel times and standard deviation of travel times are estimated based on the total trips from one MSOA to another MSOA within a one-hour start-time. The standard deviation of travel times mainly result from different delay times due to (recurring or non-recurring) congestion combined with the different (longitude and latitude coordinate based) origin-destination points of each trip from one MSOA to another. GPS data from probe vehicles (like their ride-hailing vehicles equipped with mobile phones) can provide good estimates of travel time within an urban road network (Liu and Ma, 2009; Zheng and Van, 2013), so we can use this data to represent the travel times of car commuters in London. By linking the commuter distribution information from the Census with the travel times from Uber, using the same basic zone of MSOAs, we established a daily trip dataset of car commuters.

As shown in Fig. 3, car commuting trips mainly occurred within Outer London, with local employment centers forming the main destinations. London Heathrow Airport and its surrounding built area attract the heaviest car commuting flow, which implies a higher employment attraction in these zones. People living in Inner London tend to use public transport more to commute than those in Outer London. Obviously, central London still is the largest employment center. Maybe due to the relatively high levels of public transport provision combined with high parking and congestion charge costs, most commuters, whether from Outer London or Inner London, take public transport (e.g., underground, bus, railway) to central London, and only a fraction of commuters (who tend to live in traditional wealthy areas) drive to central London. For those not commuting to central London, overall, the private car prevails in the outer

${ }^{4}$ https://movement.uber.com/?lang=en-GB 
built area. Public transport plays a role connecting the outer employment centers to the city center, as well as providing for commuters travelling within Inner London. From a temporal perspective, the average travel time is 13.9 minutes for car commuting trips in Outer London, while commuters driving to workplaces in central London spend 27.5 minutes on average getting to work, nearly double that of the former despite travelling similar distances, as shown in Fig. 4.

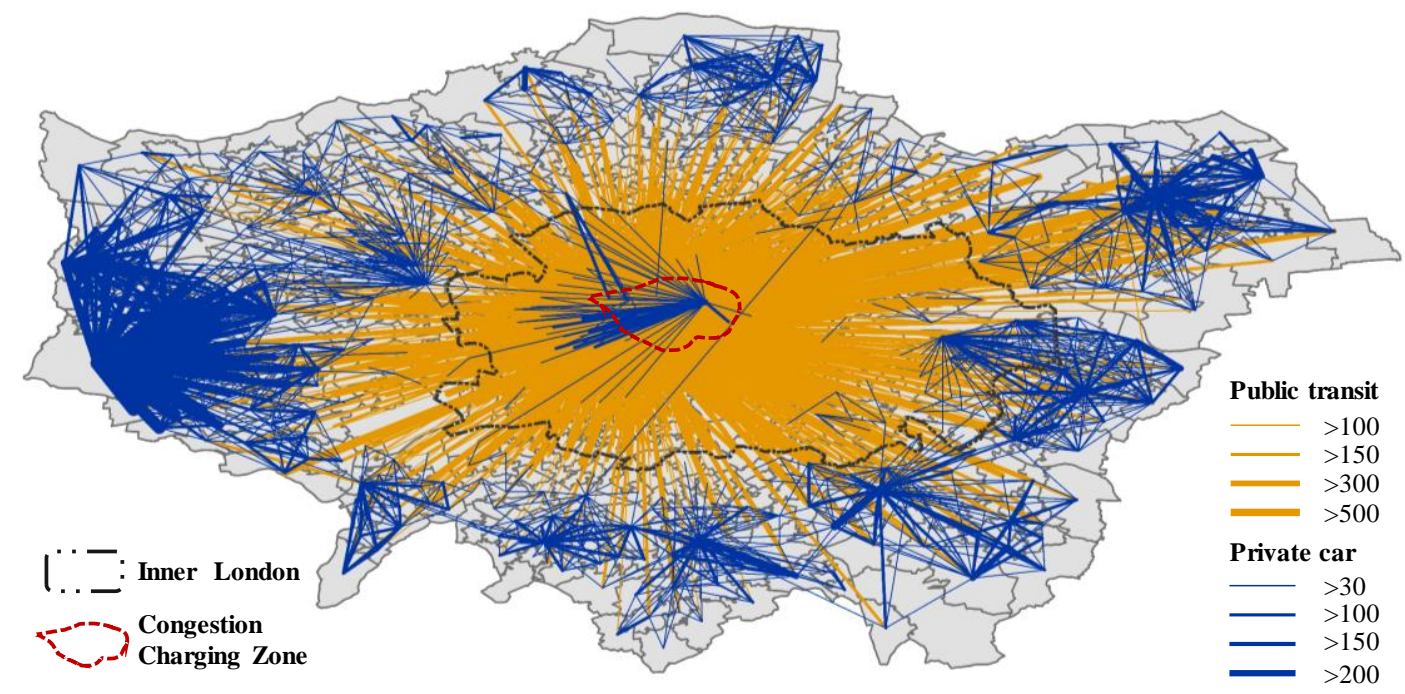

Fig. 3 The spatial distribution of commuting flows by public transit (showing flows with more than 100 trips per day) and private car (showing flows with more than 30 trips per day) in London

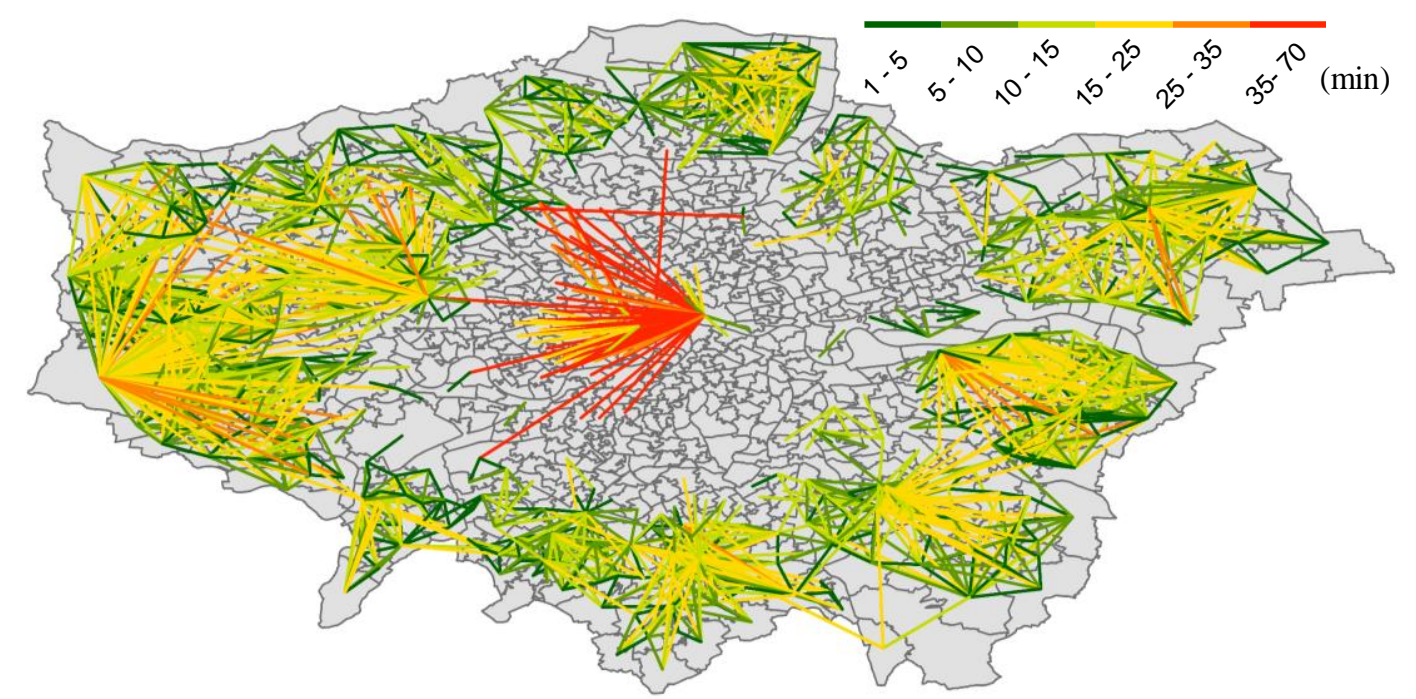

Fig. 4 Average travel time of commuting flows by car (showing flows with more than 30 trips per day) based on MSOAs.

\subsubsection{Estimating trip start-time and travel time distributions}

Based on our dataset, we can take the same or neighboring MSOAs as origindestination communities of carpooling trips. However, the Census 2011 does not include data on trip start-time of commuters, hence we need to allocate a start time. We do this using the start-time distribution of commuting trips taken from a local travel survey - the LTDS. According to the LTDS (see Fig. 5), over half of commuters depart 
within morning peak hours (7:00 am-9:00 am), and these are roughly equally divided between the first and second hours of the peak period. The numbers of commuters travelling from each origin outside of the peak hours, are likely to be too low to enable successful matching; thus, we focus only on home-work trips made during the morning peak. To guarantee matching feasibility within the carpooling service, we only consider origin-destination zone pairs with at least 24 trips recorded in the Census. For each extracted O-D pair, we assumed half of the trips were made within the AM peak; we then evenly assigned these trips to the 12 10-minute start time windows from 7:00 to 9:00. As a result, there are 66,450 morning commuting trips by car per day in our initial dataset; most of trips commence in Outer London; only 2192 trips originate within the Congestion Charge Zone (CCZ) of central London.

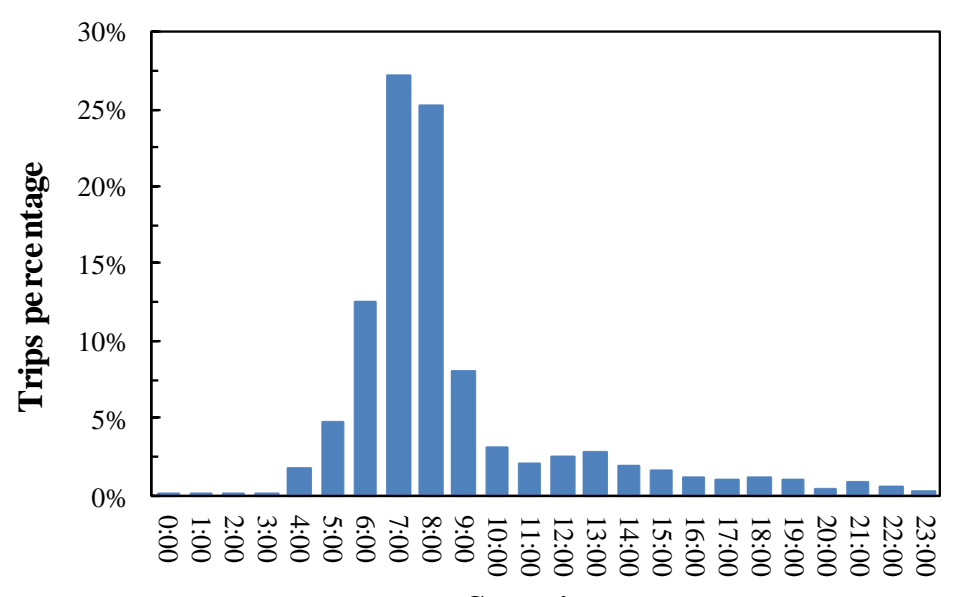

Start time

Fig. 5 Start time distribution of commuting trips by car in London

For car commuting trips in London, the average travel time is 14.4 minutes with a standard deviation of 5.2 minutes. Considering the road travel times are known to closely follow a Gamma distribution (Polus, 1979; Nie at al., 2012), it is assumed that car commuting trip travel times for each origin-destination MSOA pair follow the Gamma distribution $X \sim \Gamma(\alpha, \beta)$; the distribution parameters $\alpha$ and $\beta$ can be calculated using the mean and standard deviation of travel times in our dataset. Then we assigned each trip of each origin-destination MSOA pair with stochastic travel times based on this distribution function. In this way, the impact of different delay times and coordinate-based origin-destination locations on travel times of trips from one MSOA to another MSOA are taken into account, and can be considered to accord with real travel times. Taking the origin-destination pair with the most commuting trips as an example, there are 143 car commuting trips per day from Hounslow (MSOA 499) to Hillingdon (MSOA 479) with a mean travel time 8.97 minutes and standard deviation of 3.52 minutes. Based on the Gamma distribution $X \sim \Gamma(6.5,1.4)$, we obtain the travel time distribution shown in Fig. 6, where $80 \%$ of commuting trips have a travel time of 6 to 13 minutes. The difference between the longest commuting time and the shortest commuting time for this O-D pair is 15 minutes. 


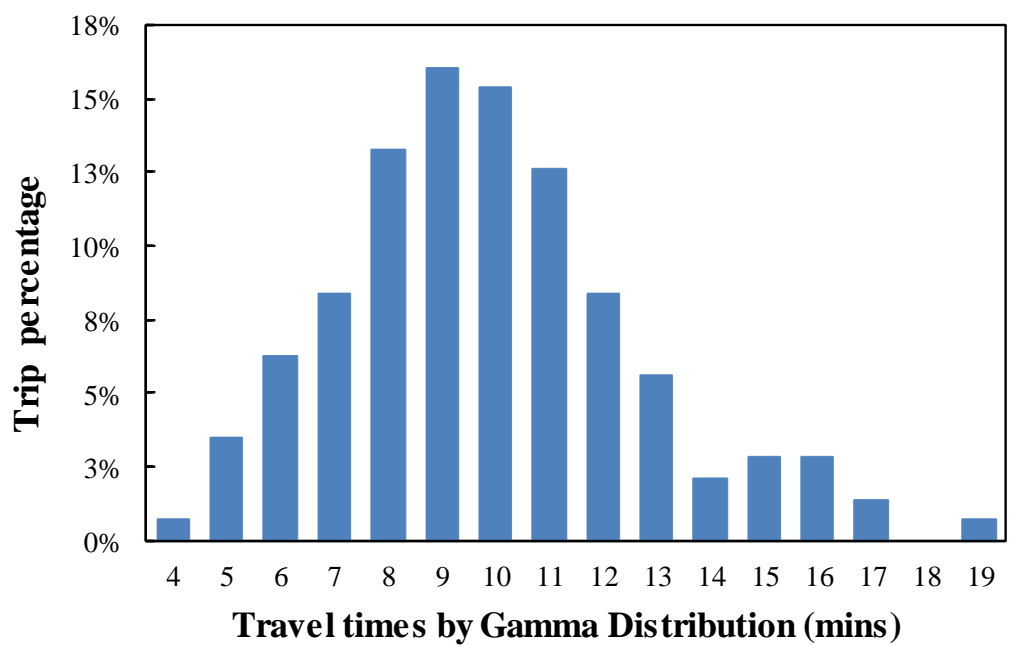

Fig. 6 Estimated travel time distribution of car commuting trips from MSOA 499 to MSOA 479

\subsubsection{Estimation of walking time, shared trip distance and trip deviation}

Taking trips from and to neighboring communities as an instance, Fig. 7 illustrates the passenger-to-driver matching mechanism of this private-car based carpooling mode. In theory, the shortest walking distance for a rider is the vertical dimension from his or her origin point (i.e. residence) to the driver's route vector (the yellow line), hence the nearest pick-up point for this rider shall be the intersection point (foot of perpendicular) of the auxiliary vertical line (the black solid line) and driver's route vector. It is likewise when this rider walks from drop-off point to their workplace. The shortest walking time can be estimated by the route deviation degree and the walking time between the driver's and the rider's respective residence (workplace). The route deviation degree is measured by the angle $\theta$ ( $\partial$ ) between the carpooling (driving) route vector and the neighbor route vector (the black dashed line) from the driver's house (workplace) community to the rider's house (workplace) community. In general, if the travelling distances from passengers to a driver are similar, larger deviation degrees would create greater detour efforts for passengers. To improve the accuracy of estimation, we used actual driving time from driver's home (workplace) MSOA to the rider's home (workplace) MSOA from our dataset as an intermediary to estimating the shortest walking time, converting driving time to walking time using average driving and walking speeds respectively. 


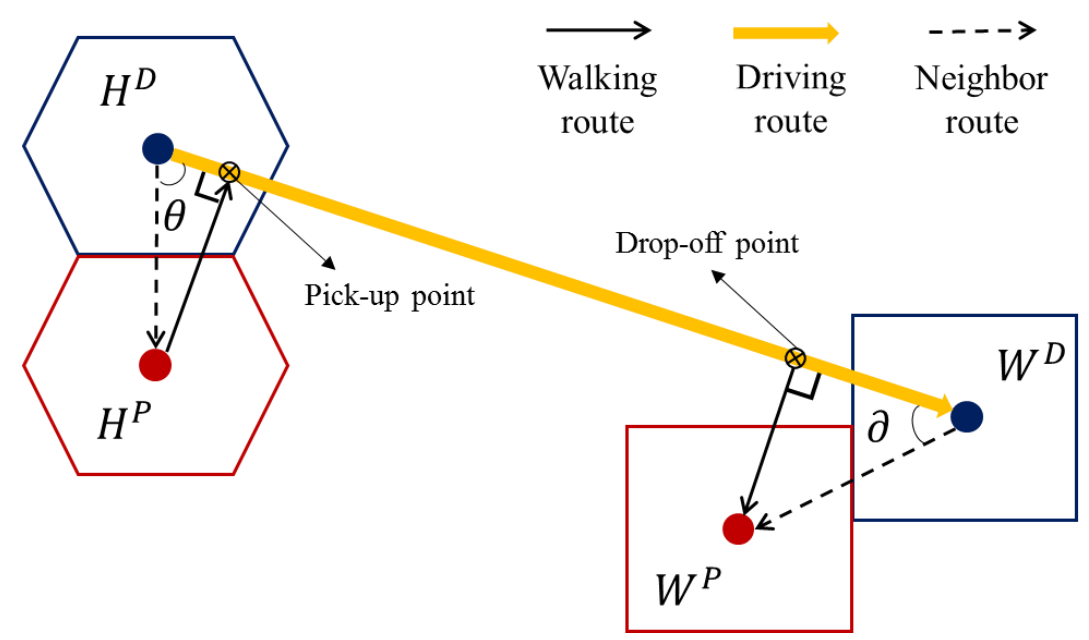

Fig. 7 Passenger-to-driver matching mechanism of private-car based carpooling for drivers and riders in neighboring communities

In Fig. 8, considering various ride-sharing patterns, we propose a route deviation angle-based method to estimate the walking time of carpooling riders from home to pick-up point, and from drop-off point to workplace. To be more specific, (a) a samecommunity shared pattern means candidates are within the same origin and destination communities, and walking time is estimated as a function of the area of communities; (b) an inclusive shared pattern means the location of a rider's residence is just on (or very close to) a driver's route. In other words, a driver's route includes a rider's original route, so no (or hardly any) walking detour is necessary; (c) a passenger-detour partial shared pattern occurs when a rider needs to walk to reach the pick-up point and then shares part of the driver's trip. The walking time can be estimated from the degree of route deviation and from the driver's home to the rider's home, shown in Fig. 8(c); (d) a passenger-detour overall shared pattern occurs when a rider and driver are from different communities and the driver's home is the pick-up point. The route deviation, or walking time, and route path can be estimated as straightline paths using the longitude and latitude of the origin and destination points. Where exact location of the home and workplace are unknown the centroid of the origin and destination zones can be used. $T_{P \rightarrow D}^{H}$ is the driving time from a driver's home to a passenger's home and $\varphi$ is the conversion factor from driving time to walking time. The proportion of the driver's trip that is shared $\omega$ can be derived as Eq. 26, where $T_{P \rightarrow D}^{W}$ is the travel time from a driver's workplace to a passenger's workplace. With carpoolers from or to the same zones the ratio of shared trip stage $\omega$ can be estimated as a function of the area of origin and destination communities. Similarly, the ratio of pick-up trip stage $\eta$ is defined in Eq. 27, for carpoolers from different communities and for carpoolers from identical communities respectively. 
655

656

657

658

659

660

661

662

663

664

665

666

667

668

669

670

671

672

673

674

675

676

677

678

679

680

681

682

683
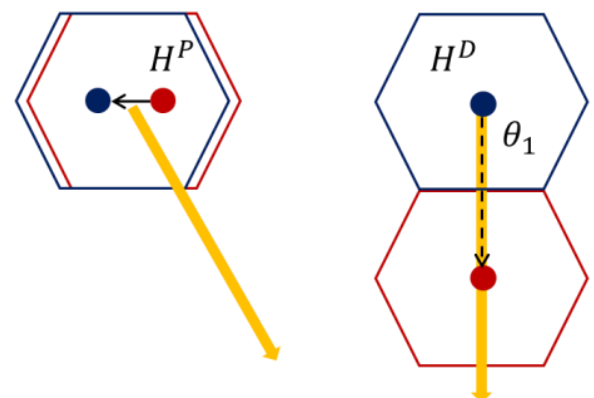

(a)

(b)

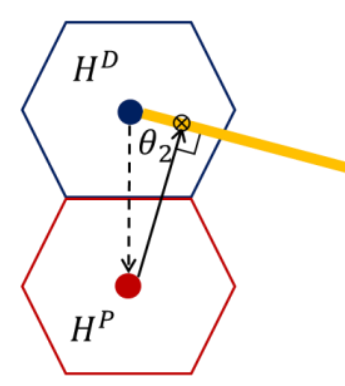

(c)

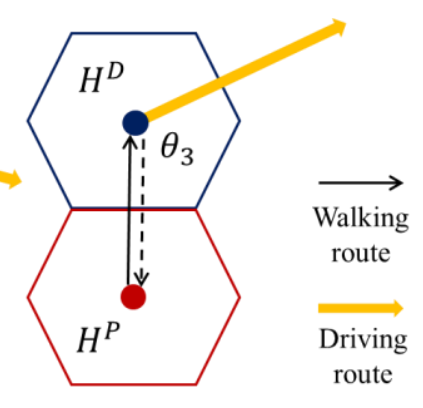

(d)

Fig. 8 The estimation of riders' walking time at origin points under different shared patterns. (a) same-community shared pattern from and to same community, average walking time is related to the community area $\overline{T_{w}^{H}}=f($ Area $)$, likewise at destination points; (b) inclusive shared pattern, $\theta_{1} \approx 0^{\circ}$ and $T_{w}^{H} \approx 0$, likewise at destination communities; (c) passenger-detour partial shared pattern, $\left|\theta_{2}\right| \leq 90^{\circ}$ and $T_{w}^{H}=\left|\sin \theta_{2}\right| \varphi T_{P \rightarrow D}^{H}$, the degree of route deviation at destination communities can be any condition, vice versa; (d) passenger-detour overall shared pattern from neighboring communities, $\left|\theta_{3}\right|>90^{\circ}$ and $T_{w}^{H}=\varphi T_{P \rightarrow D}^{H}$, likewise at destination communities. Note that $\theta \in\left(-180^{\circ}, 180^{\circ}\right)$.

$\omega=1-\left(\max \{\cos \theta, 0\} \cdot T_{P \rightarrow D}^{H}+\max \{\cos \partial, 0\} \cdot T_{P \rightarrow D}^{W}\right) / T_{t}^{D}$

$\eta=\max \{\cos \theta, 0\} \cdot T_{P \rightarrow D}^{H} / T_{t}^{D}$ or $\eta=\overline{T_{w}^{H}} / \varphi T_{t}^{D}$

\subsubsection{Parameter setting}

Referring to the results in previous works (Gardner and Abraham, 2007; Abrantes and Wardman, 2011), we take the numerical magnitude relation for involved unit cost factors as $\varepsilon^{-} \leq \delta^{-} \leq \delta^{+} \leq \lambda \leq \alpha \leq \gamma \leq \varepsilon^{+}$. Focusing on car commuters in London, we take $£ 0.10$ /minute as the value of IVT at 2011 prices and incomes and take the other unit cost factors in the form of time multipliers as $\varepsilon^{+}=2.8, \gamma=1.7, \alpha=1.4, \lambda=1$, $\delta^{+}=0.7, \delta^{-}=0.6, \varepsilon^{-}=0.5$ (Abrantes and Wardman, 2011). Note that the unit cost factor of the sharing psychological penalty takes a value between the unit cost of walking time and the unit cost of IVT. Moreover, lack of empirical operating data and specific preferences of carpoolers, we assume all participants in this carpooling system are strangers and share their trips for the first time, hence we take $A L^{\prime}=0, M T=1, \quad \kappa=1.1$ in the estimation of psychological penalty cost.

For the monetary trip cost, parking fees in Outer London are an average $£ 5$ per day based on the price of a season-ticket for National Car Parks (NCP) ${ }^{5}$. In addition, an $£ 11.50$ daily charge is payable when driving within the Congestion Charging Zone (CCZ) from 07:00 to18:00 on a weekday, however those living within or immediately

\footnotetext{
${ }^{5}$ https://www.ncp.co.uk/parking-solutions/season-tickets/
} 
684

685

686

687

688

689

690

691

692

693

694

695

696

697

698

699

700

701

702

703

704

705

706

707

708

709

710

711

712

713

714

715

716

adjacent to the CCZ enjoy a 90\% residents' discount. According to the LTDS in 2017 (TfL, 2018), the average travel speed within Outer London during morning peak hours is about $30 \mathrm{~km} /$ hour. Considering average new car fuel consumption and the proportions of petrol to diesel cars, as well as the fuel prices from 2011 in $\mathrm{UK}^{6}$, the unit fuel cost is set as $£ 0.04 / \mathrm{min}$ on average. The other variable trip costs including wear and tear costs, insurance costs and so on are about $£ 0.1 / \mathrm{min}$ (Danish Ministry of Transport, 2013). It is assumed the carpooling platform will charge a service fee accounting for $10 \%$ of cost savings of the rider and the driver from each successful carpooling trip; this level of service fee will not significantly affect the carpooling matching rate.

For the parameters used in the estimation of carbon emission costs, carbon dioxide emissions (total $\mathrm{CO}_{2}$ equivalent) are $0.14 \mathrm{~kg} / \mathrm{km}$ on average from cars that were new between 2007 to $2016^{7}$. Non-traded emissions are assigned a value of about $£ 64 /$ metric ton $\mathrm{CO}_{2}$ equivalent in 2016 (Rosenow et al., 2018). After conversion, the unit cost of carbon emissions is $£ 0.0045 / \mathrm{min}$. The impact factor of the additional passenger's weight on fuel consumption is about $4.5 \times 10^{-3} \mathrm{liter} / 100 \mathrm{~km} / \mathrm{kg}$ as reported by the US Environmental Protection Agency (EPA, 2016). Based on the average weight of London adult residents ${ }^{8}$, the fuel-using cost factor of each additional carpooling passenger $\beta_{1}$ is $£ 0.0023 / \mathrm{min} /$ person (see Eq. 2) and the carbon emissions factor of each additional carpooling passenger $\beta_{2}$ is $0.046 /$ person (Eq. 8 and Eq. 9).

Regarding the parameters of walking time estimation, the mean walking speed used in TFL's Public Transport Accessibility Levels (PTALs) ${ }^{9}$ is $80 \mathrm{~m} / \mathrm{min}$; we take $100 \mathrm{~m} / \mathrm{min}$ as the input factor of walking speed due to commuters frequently quickening their pace (Galiza et al., 2011). As data on walking times between MSOAs was not available, we used a conversion factor applied to drive time to get an estimated walking time based on travel speed, that is $\varphi=5$. For matched pairs with a same-community shared pattern, we assume that MSOAs approximate a circle and the origin or destination points of two matched trips are distributed randomly within the MSOA. The probability density for the distance $l$ between two random points in a circle of radius $r$ is given by García (2005) as Eq. 28. Hence, the expected value of this distance is derived in Eq. 29. Therefore, average walking distance $d_{w}$ is a function of the community area (unit is $\mathrm{km}^{2}$ ); thus the average walking time within a MSOA is $\overline{T_{w}} \approx 5 \sqrt{\text { Area }}$ minutes based on the walking speed.

$p(l)=\frac{4 l}{\pi} \arccos \frac{l}{2 r}-\frac{2 l^{2}}{\pi r^{4}} \sqrt{r^{2}-\frac{l^{2}}{4}}$

${ }^{6}$ https://www.gov.uk/government/statistical-data-sets/energy-and-environment-datatables-env

7 https://www.smmt.co.uk/reports/co2-report/

8 https://data.london.gov.uk/dataset/obesity-adults

${ }_{9}$ https://data.london.gov.uk/dataset/public-transport-accessibility-levels 
Unless otherwise stated, $U_{s}=25 \mathrm{~min}, U_{w}=10 \mathrm{~min}$, and the role ratio of driver and passenger is 1 to 1 with no flexible roles. All experiments were run on a computer with an Intel (R) Core(TM) i5-3320M 2.60 GHz CPU and a 8GB RAM. The carpooling matching model in this paper was implemented in C\# 2015 and was solved by a commercial optimization software package, IBM ILOG CPLEX (version 12.8). The CPU time for computing this matching model involving 66,450 trips is within two minutes.

\section{Results}

\subsection{Basic results}

Based on the initial parameters and dataset, we obtain the optimal solutions of the matching model involving 66,450 car commuting trips between 3243 OD pairs. First, 12,733 carpooling trips can be formed in total with the matching rate $38.3 \%$, generalized cost saving rate $8.9 \%$ and carbon reduction rate $19.4 \%$. Second, for matched carpooling trips, both the driver and passenger in a carpool together can save $£ 3.3$ per trip accounting for $21 \%$ of total trip cost. Riders can save $£ 1.5$ per trip, slightly less than drivers' savings. The carpooling platform can earn about $£ 5000$ per workday from service fee. The carbon emission reduction benefit is $£ 0.06$ on average with a reduction rate up to 56\%; in total, carpooling trips can save 11.8 metric tons $\mathrm{CO}_{2}$ equivalent emissions per day. Third, for the 2192 trips within Congestion Charge zone (150 carpoolers are estimated to benefit from the residents' discount), the matching performance is better with a matching rate of $76.9 \%$ and a cost saving rate of $21.3 \%$. Fourth, the cost-based carpooling equity factor is 0.4 on average. The $10 \%$ of the lowest and the highest in the equity factor distribution are 0.05 and 0.79 on average, respectively, showing obvious gaps among cost savings of individual trips. From the perspective of travel times, about two thirds of riders need to spend more time travelling in total (including the walking stages) than when they were driving-alone. The increased travel time is $27.5 \%$ greater than the original driving-alone travel time on average. To guarantee social fairness in this carpooling model, it may be necessary to adjust the fee-sharing ratios between drivers and riders or to consider cost-based dynamic pricing for the carpooling fee.

We estimated separate matching result statistics for each of the sharing patterns illustrated in Fig. 8. Considering there is scarcely any totally inclusive trips (i.e. $|\theta|=0$ and $\left.|\partial|=0^{\circ}\right)$ in practice, we assume that trips with a very small degree of route deviation (here we take $|\theta| \leq 5^{\circ}$ and $|\partial| \leq 5^{\circ}$ ) are inclusive shared pattern trips to explore the associated matching performance. As shown in Table. 2, over half of the carpooling trips require a detour. Inclusive shared pattern trips have the highest cost savings, $£ 4.55$ per trip ( $30 \%$ of the trip cost), and involve the least walking time of around 1 min per trip. The passenger-detour overall shared pattern trips provided the 
greatest reduction in carbon emissions at $70 \%$, but riders in this category have to walk 7 minutes to their pick-up points or workplaces on average. In general, a higher additional walking time created in the matching process reduces generalized cost savings but results in greater carbon emission reductions. This suggests that private-car based carpooling, with the our passenger-to-driver based matching model, could be more sustainable than ride-hailing based carpooling, because a) in this system there are no vehicle detour, but any detour is made on foot - any detour by carpooling drivers would erode the carbon savings (Liu et al., 2019); b) the carbon reduction of ridehailing based carpooling mainly derives from a second passenger, where one driver with one passenger in a private-car based carpool has a similar effect. Given the number of seats in a car is limited, private-car based carpooling has a higher potential in reducing carbon emissions. While the inclusive shared pattern without detour is the preferred matching pattern for ride-hailing based carpooling for carbon savings (Liu et al, 2019), for private-car based carpooling this pattern saves the least carbon. Note that the cost saving rates and carbon reduction rates are estimated based on the cost and carbon reduction of matched trips with corresponding shared patterns.

Table 2. Matching performances for carpooling trips with different route shared patterns

\begin{tabular}{|c|c|c|c|c|c|c|}
\hline Shared pattern & $\begin{array}{c}\text { Number } \\
\text { percent }\end{array}$ & $\begin{array}{c}\text { Cost } \\
\text { saving }(£)\end{array}$ & $\begin{array}{c}\text { Cost } \\
\text { saving } \\
\text { rate }\end{array}$ & $\begin{array}{l}\text { Carbon reduction } \\
\qquad\left(10^{-2} £\right)\end{array}$ & $\begin{array}{l}\text { Carbon } \\
\text { reduction rate }\end{array}$ & $\begin{array}{l}\text { Walking time } \\
\text { (min) }\end{array}$ \\
\hline $\begin{array}{c}\text { Same- } \\
\text { community }\end{array}$ & $36.5 \%$ & 2.95 & $18.8 \%$ & 7.26 & $66.4 \%$ & 5.8 \\
\hline Inclusive & $7.9 \%$ & 4.55 & $30.6 \%$ & 3.17 & $38.2 \%$ & 1.3 \\
\hline $\begin{array}{c}\text { Passenger-detour } \\
\text { partial }\end{array}$ & $53.5 \%$ & 3.33 & $21.0 \%$ & 5.37 & $50.0 \%$ & 4.8 \\
\hline $\begin{array}{c}\text { Passenger-detour } \\
\text { overall }\end{array}$ & $2.1 \%$ & 3.31 & $19.3 \%$ & 8.52 & $70.0 \%$ & 7.2 \\
\hline
\end{tabular}

In addition, we compared the additional walking time required for carpooling with those for public transport in London. The carpooling riders need to walk from their residence (drop-off point) to the pick-up point (workplace) 4.83 minutes on average, while London commuters taking the bus need to walk 4.9 minutes and those taking the underground walk 7.4 minutes (DfT, 2017b). Carpooling, in this case, requires shorter walking times than public transport, which helps demonstrate the acceptability of the proposed carpooling model.

\subsection{The impact of participant flexibility with the various participant rates}

We tested three different types of participant flexibilities in the passenger-to-driver matching model - role flexibility, walking detour tolerance, and schedule deviation 
tolerance. Participant role flexibility refers to the willingness of carpoolers to be 1) a driver, or 2) a rider, or 3) either a driver or a rider. We define the participants who choose to be 3 ) either a driver or a rider as flexible carpoolers. Walking flexibility is the willingness of riders to make a walking detour to the pick-up points or destination workplaces. Schedule deviation tolerance indicates the willingness of carpoolers accept changes to their usual departure time or arrival time in order to form a carpooling trip. The impact of low participant rates was tested by subsampling our dataset, randomly removing increasing fractions of commuters (10\% to $80 \%)$ from each MSOA.

\subsubsection{Role flexibility}

In this section, we explore the impact of the role flexibility on the matching rate with various participation rates. In the experiments, we considered two scenarios: 1) various ratios of drivers to passengers with no flexible carpoolers; 2) various ratios of flexible carpoolers to inflexible carpoolers, with the ratio of inflexible drivers to inflexible passengers set as 1:1. We computed the average matching rates and generalized cost saving rates for varying levels of system participant rate (from $20 \%$ to $100 \%$ of all car commuting trips) and role flexibilities, illustrated in Fig. 9(a) and Fig. 9(b), respectively, where the left-hand side gives results for scenario 1) and the right scenario 2). The warmer color is associated with a higher matching rate and cost saving rate.
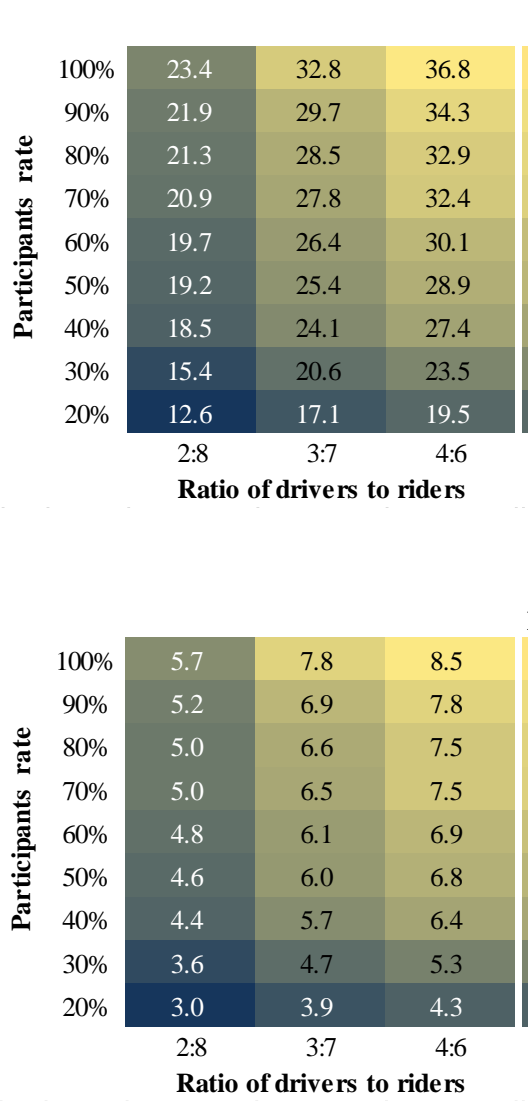

Carpooling matching rate (\%)

\begin{tabular}{|l|l|l|l|l|l|}
\hline 38.3 & 46.4 & 53.9 & 61.3 & 68.7 & 75.9 \\
\hline 35.5 & 43.5 & 51.1 & 58.3 & 65.5 & 72.7 \\
34.4 & 42.0 & 49.6 & 57.5 & 64.6 & 71.9 \\
33.6 & 41.4 & 48.8 & 56.1 & 63.4 & 70.6 \\
31.0 & 38.7 & 46.4 & 53.8 & 60.8 & 68.3 \\
30.0 & 37.1 & 44.5 & 51.7 & 58.3 & 65.9 \\
\hline 27.7 & 35.3 & 42.2 & 48.9 & 56.2 & 63.2 \\
\hline 24.1 & 30.6 & 37.2 & 43.8 & 50.5 & 57.4 \\
\hline 19.6 & 25.4 & 30.9 & 36.0 & 43.0 & 49.4 \\
\hline $0 \%$ & $20 \%$ & $40 \%$ & $60 \%$ & $80 \%$ & $100 \%$ \\
& \multicolumn{7}{c}{ Ratio of flexible carpoolers } \\
\end{tabular}

(a)

Mean generalized cost saving rate $(\%)$

\begin{tabular}{|c|c|c|c|c|c|}
\hline 8.8 & 10.8 & 12.6 & 14.4 & 16.4 & 18.3 \\
\hline 8.1 & 10.0 & 11.8 & 13.6 & 15.3 & 17.2 \\
\hline 7.8 & 9.6 & 11.4 & 13.2 & 15.0 & 16.8 \\
\hline 7.7 & 9.5 & 11.3 & 13.1 & 14.9 & 16.7 \\
\hline 7.2 & 8.9 & 10.7 & 12.5 & 14.2 & 16.0 \\
\hline 6.9 & 8.6 & 10.4 & 12.2 & 13.8 & 15.7 \\
\hline 6.5 & 8.2 & 9.8 & 11.5 & 13.3 & 15.0 \\
\hline 5.4 & 6.9 & 8.6 & 10.0 & 11.6 & 13.2 \\
\hline 4.3 & 5.7 & 7.1 & 8.2 & 9.7 & 11.3 \\
\hline $0 \%$ & $20 \%$ & $40 \%$ & $60 \%$ & $80 \%$ & $100 \%$ \\
\hline
\end{tabular}


Fig. 9 The impact of role flexibility of carpooling users on matching rate (a) and generalized cost saving rate (b) with varying participant rates.

The results show, as expected, that for a given level of role flexibility, both the average matching rate and cost saving rate increases with the number of trips in the system. These two indexes show a sharp decline when the ratio of candidates willing to carpool dropping from $40 \%$ to $20 \%$, therefore, it is beneficial to reach a moderate participant level before launching the carpooling service. Similarly, for a given participant ratio, the matching system performs better with the higher level of role flexibility, but the marginal increases diminish, especially under scenario a).

We also find that additional participant carpoolers are more beneficial at low ratios of flexible carpoolers, while higher ratios of flexible carpoolers are more beneficial at low participant rates, which is not significant in the scenario 1). For instance, Fig. 9(a) shows that increasing the ratio of flexible carpoolers from $20 \%$ to $100 \%$ causes the average matching rate to double with a $20 \%$ participant rate and a $64 \%$ increase when all trips are available.

The results suggest that low role flexibility can heavily limit the ability of the system to establish matches. Even with a $100 \%$ participant rate, the average matching rate is only $23.4 \%$ when $80 \%$ of participants choose the single role of drivers and $20 \%$ of participants choose the single role of riders. With fewer participants, the severe imbalance between drivers and riders can result in a match rate of less than $15 \%$ and a cost saving rate of less than $5 \%$. The reason for this is that more drivers than riders in the carpooling system limits the number of ride-sharing opportunities and consequently also the number of matches that are established, and vice versa. A higher role flexibility, on the other hand, can make up for a lack of density. For example, if all carpoolers choose the flexible role, nearly half of carpooling trips can be shared at the lowest density. Therefore, it is important for carpooling providers to encourage more users to be flexible carpoolers and to keep the role balance of candidate drivers and riders.

\subsubsection{Walk detour flexibility}

In this section, we investigate the results of an experiment designed to quantify the effects of walk detour flexibility on system performance. Similar to the previous section, we compute the matching rates and cost saving rates for various levels of system density and vary the walking time thresholds of riders (from 4 minutes to 12 minutes), as illustrated in Fig. 10. Note that the mix of participant types here is back to the initial scenarios, that is the same ratio of drivers to passengers with no flexible roles. The results show, again, that for a given participant rate, the average matching rate and cost saving rate increases with higher walking time thresholds in the system, but the marginal increases dramatically diminish. It is also apparent that for a given detour flexibility, additional density has a greater impact on the matching rate especially when the ratio of candidates willing to carpool is at a lower level. 


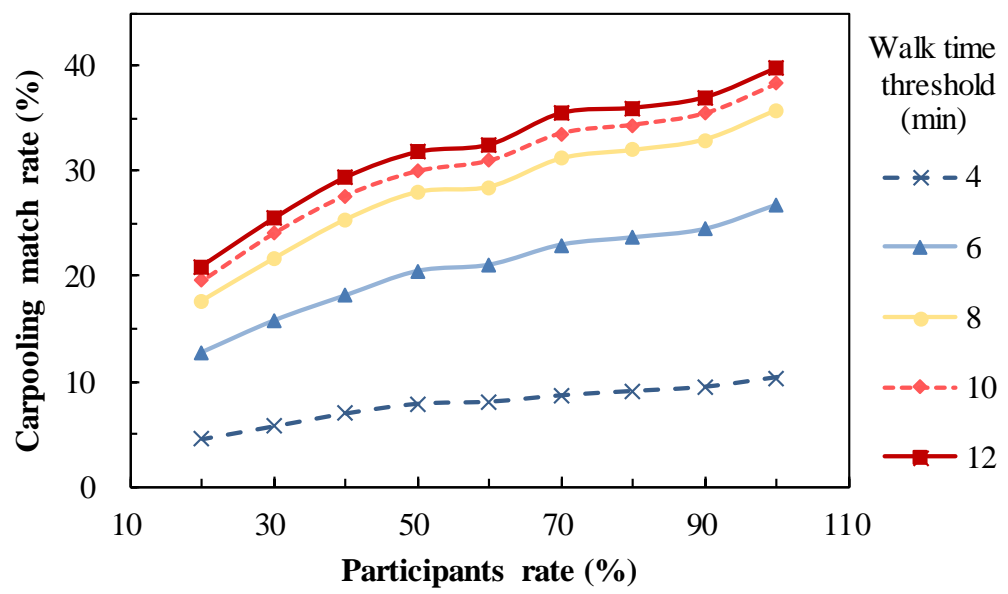

(a)

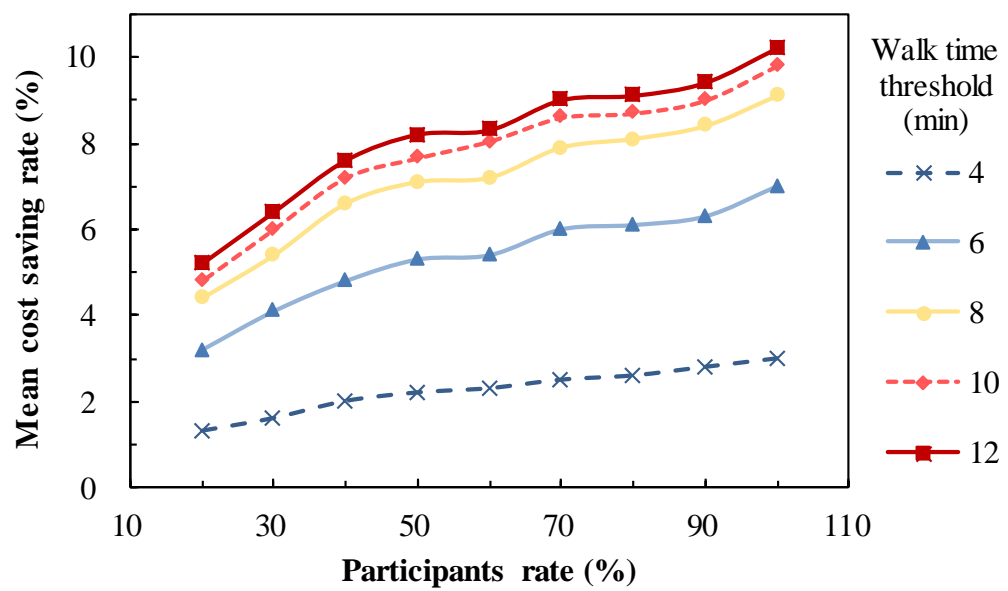

(b)

Fig. 10 The impact of walking detour flexibility of riders on match rate (a) and generalized cost saving rate (b) with varying participant rates.

We observe large gaps between settings with high and low detour flexibility. Apparently, the willingness of riders to walk a longer time can increase the matching rate substantially. It also appears that increasing detour flexibility from lower values of 4 minutes to moderate values of 8 minutes, contributes to significant increases in the matching rate even at high system densities, while the system does not gain much if walk time threshold is changed from moderate values of 8 minutes to higher values of 12 minutes. Based on these results, we suggest a two-pronged approach to improve the matching performance: a) the operators can set a moderate walk time threshold based on a local preliminary survey when designing the system and, b) the policy-makers could advertise the benefits of active travel and improve walking environments to encourage riders to walk more in practice.

\subsubsection{Schedule deviation tolerance flexibility}

In this section, we present the results of an experiment designed to analyze the impact of schedule deviation tolerance flexibility of riders on the system matching rate.

Taking the tolerance degree on arriving later as an example, we explore the impact of 
the various levels of arriving later penalty factors (from $£ 0.05 / \mathrm{min}$ to $£ 0.50 / \mathrm{min}$ penalty cost factor) and vary the system participant rate. The higher penalty factor indicates a lower arriving later tolerance flexibility. Recall from Section 3.5.4 that we take the initial arriving later penalty factor as a 2.8 multiplier of IVT, namely $£ 0.28 / \mathrm{min}$.

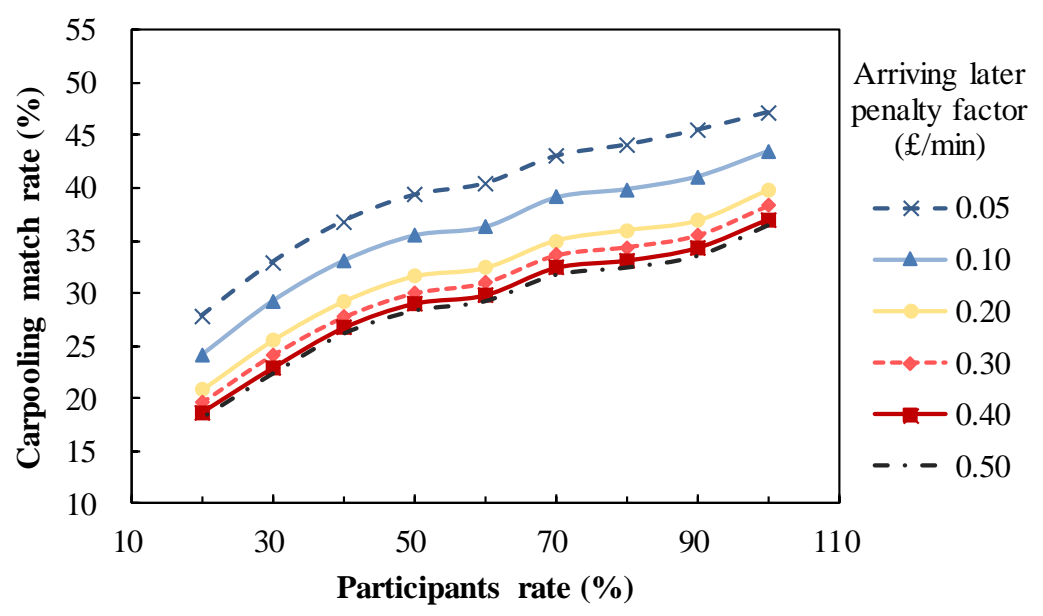

Fig. 11 The impact of arriving later tolerance flexibility of riders on matching rate with varying participant rates.

Fig. 11 shows that for a given penalty level, a similar rapid decline of matching rate with decreasing participant rate at the lower level from $40 \%$ to $20 \%$, which demonstrates the importance of sufficient initial carpooling participants again. Matching performance drops remarkably when the penalty factor increases from 0.05 to 0.2 , while the lower flexibility for late arrival reduces the matching rate slightly, the trend of which is opposite to matching performances of previous experiments. The associated reason may be that the moderate level of the penalty factor has eliminated most of potential carpooling trips that would result in carpoolers being late to work. Without eroding the matching rate much, we can set a relatively higher schedule deviation penalty factor to guarantee the time reliability of carpooling services. In contrast to the previous participant role flexibilities and detour flexibilities, the results show that flexibility in tolerance of arriving late has less impact on the functioning of this carpooling system. Even with the highest penalty factor, the system can maintain a $20 \%$ matching rate at the lowest carpooling participant rate. There may be two reasons, 1) the time duration of arriving later for work is relatively small, that is 2.5 minutes on average in the initial matching system; thus the impact of a higher penalty factor for arriving later is limited; 2) a lower schedule deviation tolerance flexibility can only reduce the trip cost savings and then damage the matching rate indirectly; while a lower role flexibility and lower walk time threshold can exclude considerable numbers of candidates directly from our matching system, based on the set of feasible carpooling trips.

\subsubsection{Market access flexibility}


In Fig. 12, we compute the matching rates and carbon emission reductions for various levels of market access limitations (market access threshold $\mathrm{N}$ from 6 to 12). Only if an MSOA generates more than $\mathrm{N}$ car commuting trips, would it have a chance of being included in the local carpooling service market. Results show that if we include more MSOAs in the carpooling system, the matching rates change slightly, while the carbon emission reductions rise remarkably. At $\mathrm{N}=6$, carpooling trips can save $£ 1421$ of carbon per day, that is a reduction of more than 20 metric tons of $\mathrm{CO}_{2}$ equivalent emissions.

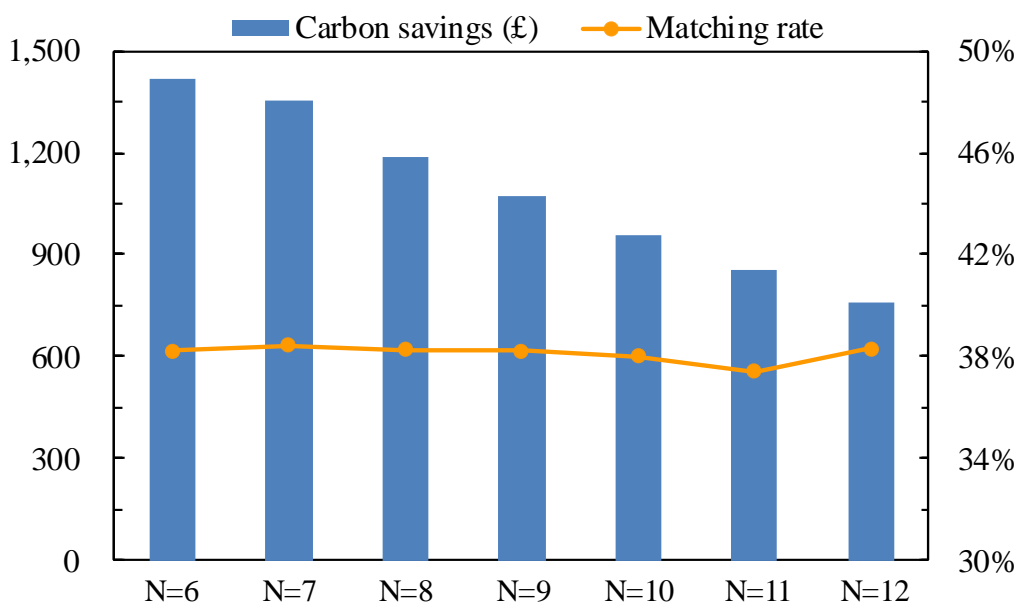

Fig. 12 The effects of introducing more MSOAs into the matching system on matching rate and carbon reduction. The market access threshold $\mathrm{N}$ means the lowest number of car trips within AM peak hours for a MSOA to be included in the carpooling match system.

\subsection{The impact of penalty cost of riders with diverse cost-sharing ratios}

In this section, we investigate the impact of the additional penalty of riders due to ridesharing on the matching performance at various fee pricing levels. In our passengerto-driver matching mode, a driver controls the whole trip and would not detour to pick up or set off riders, while riders need to not only bear the sharing psychological cost including the additional perceived control loss, but also pay an additional walking effort cost. Taking a certain cost-sharing ratio between drivers and passengers as the carpooling fee pricing rule, we compute the average matching rates and cost saving rates for various levels of cost-sharing ratios (riders sharing $20 \%-70 \%$ of a carpooling trip's driving cost) and vary the related penalty factor of riders in the system. We explore the impact of two aspects of penalty costs: the sharing psychological loss of riders (one third of the initial value to four times the initial value), the walking effort loss of riders (one third of the initial value to three times the initial value), shown in Fig. 13 and Fig. 14. Note that we introduce an amplification factor for the additional control loss of riders into the rider's psychological loss to distinguish it from the driver's psychological loss. Recall from Section 3.5.4 that we take the initial psychological penalty factor, walking penalty factor and the amplification factor of rider as $£ 0.14 / \mathrm{min}, £ 0.17 / \mathrm{min}$ and 1.1 , respectively. 


\subsubsection{Sharing psychological penalty}

The results in Fig. 13 show that for any given ratio of fee-sharing (riders sharing $20 \%-70 \%$ of a carpooling trip's driving cost), both the average matching rate and cost saving rate increase when the penalty factor reduces. On the contrary, a higher sensitivity to sharing psychological loss can heavily limit the ability of the system to form matches. A sharp rise in matching performance occurs at moderate penalty levels, and the marginal increase diminishes at higher penalty levels. Setting an appropriate psychological penalty factor can reduce the psychological loss while sacrificing fewer carpooling trips. For various levels of sharing psychological sensitivity, as expected, the optimal fee-sharing ratio for trip matching rates is not the initial half to half, but between $30 \%$ and $40 \%$ for passengers. The cost savings of riders and drivers are approximately equal (the carpooling equity factor is 0.51 ) at $40 \%$ fee-sharing ratio. It is necessary to investigate the optimal fee-sharing ratio based on the specific matching mode to achieve a better matching performance and greater social fairness for carpooling. Moreover, when the psychological penalty factor increases to a higher level, with riders paying $30 \%$ of the carpooling fee, this can result in the highest matching rates, but paying $40 \%$ would create the highest cost saving rate. This slight difference between the two matching indexes may be because the additional control loss of a rider becomes less significant when all carpoolers show a high sensitivity to the psychological loss, then the fee-sharing difference between the rider and the driver will also shrink.

\begin{tabular}{|c|c|c|c|c|c|c|}
\hline \multirow[b]{2}{*}{0.7} & \multicolumn{6}{|c|}{ Carpooling match rate (\%) } \\
\hline & 36.7 & 33.4 & 25.9 & 16.8 & 12.5 & 10.2 \\
\hline 0.6 & 45.5 & 41.8 & 32.0 & 20.9 & 15.6 & 12.8 \\
\hline 0.5 & 53.7 & 49.7 & 38.3 & 24.4 & 18.5 & 15.4 \\
\hline 0.4 & 59.1 & 55.5 & 42.7 & 26.9 & 20.6 & 17.2 \\
\hline 0.3 & 61.9 & 57.9 & 43.1 & 27.1 & 20.8 & 17.4 \\
\hline 0.2 & 61.7 & 55.9 & 38.1 & 23.6 & 18.0 & 14.6 \\
\hline
\end{tabular}

Psychological cost factor of rider and driver $\left(10^{-2} £ / \mathrm{min}\right)$

(a)

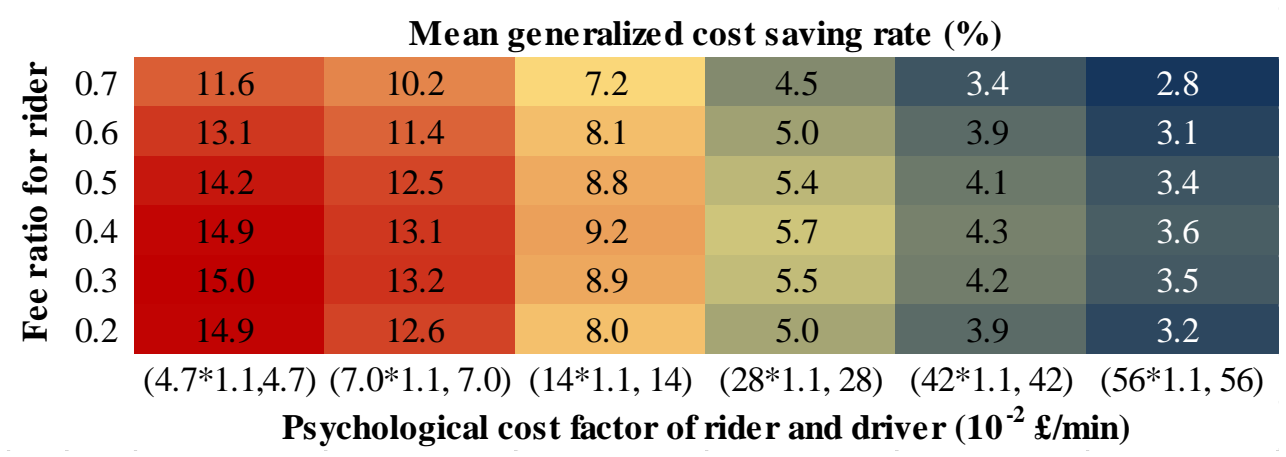

(b) 
Fig. 13 The impact of psychological penalty considering the additional control loss of riders on match rate (a) and generalized cost saving rate (b) with diverse fee-sharing ratios

\subsubsection{Walk effort penalty}

Fig. 14 shows that for any one given ratio of fee-sharing (riders sharing 20\%-70\% of a carpooling trip's driving cost), the matching performances become better with a lower level of walking penalty factor, but the marginal increases diminish. In contrast to the psychological penalty, the results show that the walking effort factor has greater impact on the functioning of this carpooling system. If the penalty factor increases to a higher level, the matching rates can go down to $10 \%$ with a cost saving rate of less than $3 \%$. Measures to improve the attitude of carpoolers to active trips like walking and to reduce the walk-effort sensitivity could significantly facilitate the development of carpooling services. Once again, the best matching rates and carpooling equities are not derived from pricing the fee-sharing evenly. If the walking effort penalty factor is at a low level, riders should pay $40 \%$ of the fee in order to obtain better matching performances. If the penalty factor rises, riders should pay less money since they have already paid more physical effort for their carpooling trip.

\begin{tabular}{|c|c|c|c|c|c|}
\hline \multirow{2}{*}{0.7} & \multicolumn{5}{|c|}{ Carpooling match rate (\%) } \\
\hline & 42.6 & 37.7 & 25.9 & 11.8 & 6.5 \\
\hline 0.6 & 49.2 & 45.0 & 32.0 & 15.6 & 8.4 \\
\hline 0.5 & 53.0 & 50.0 & 38.3 & 19.8 & 10.5 \\
\hline 0.4 & 53.6 & 51.6 & 42.7 & 23.7 & 12.9 \\
\hline 0.3 & 50.2 & 48.7 & 43.1 & 26.1 & 14.9 \\
\hline .2 & 42.0 & 41.2 & 38.1 & 25.8 & 15.8 \\
\hline & 5.7 & 8.5 & 17 & 34 & 51 \\
\hline
\end{tabular}

Walk-effort factor of rider and driver $\left(10^{-2} £ / m i n\right)$

(a)

\begin{tabular}{|c|c|c|c|c|c|}
\hline \multirow[b]{2}{*}{0.7} & \multicolumn{5}{|c|}{ Mean gene ralize d cost saving rate (\%) } \\
\hline & 12.1 & 10.6 & 7.2 & 3.4 & 2.0 \\
\hline 0.6 & 13.0 & 11.6 & 8.1 & 4.0 & 2.3 \\
\hline & 13.2 & 12.2 & 8.8 & 4.5 & 2.5 \\
\hline 0.4 & 13.2 & 12.2 & 9.1 & 4.9 & 2.8 \\
\hline 0.3 & 12.6 & 11.6 & 8.9 & 5.0 & 2.9 \\
\hline 0.2 & 11.0 & 10.3 & 8.0 & 4.7 & 2.9 \\
\hline & 5.7 & 8.5 & 17 & 34 & 51 \\
\hline
\end{tabular}

(b)

Fig. 14 The impact of walking penalty of riders on match rate (a) and generalized cost saving rate (b) for different fee sharing ratio of riders

\subsection{The effect of carpooling promotion instruments on matching performances}

In this section, we explore the effect of some typical instruments that aim to promote car-pooling on matching performance and carbon emissions reduction. Based on previous practice and research ( $\mathrm{Su}$ and Zhou, 2012; Vanoutrive et al. 2012; 
Delhomme and Gheorghiu, 2016), popular promotion instruments include: costoriented measures, service-oriented measures and low-carbon-oriented measures. The first focuses on influencing trip costs including parking fee discounts for carpoolers (or parking surcharges for single occupancy vehicles), congestion charge exemptions and travel allowance subsidies on carpooling trips; the second seeks to improve carpooling associated services including through High Occupancy Vehicle (HOV) lanes and tailored trip information provided by carpooling and other mobility service platforms; the last focuses on carbon emissions reduction through measures such as expanding the scope of services and promoting electric vehicles in carpooling. Here we focus on the first type of measures and introduce them into our carpooling matching model to assess their specific effect.

In Fig. 15, we compute the matching performance and carbon emissions reduction for various levels of parking discounts (from 0 to $30 \%$ off for carpoolers) and parking surcharges (from 0 to an additional 30\% for single occupancy vehicles). Results show that both the parking discounts and the parking surcharges can help match more trips and reduce carbon emissions. However, parking surcharges are more effective than parking discounts, because carpoolers can save more in contrast with driving alone under a parking surcharges policy.

We also examined the impact of a travel cost subsidy for carpoolers. The level of subsidy is trip-specific and based on the actual carbon emissions reduction achieved for each carpooling trip. This choice is based on the findings from previous research (Liu et al., 2019), which showed that optimal emissions reduction can only be achieved with a trip-specific model for trip subsidies. However, in the case of London, these trip subsidies scarcely improve matching rates and carbon reductions due to today's low carbon value $(£ 0.064 / \mathrm{kg})$. If the recent trend of increasing carbon trading prices continues, the effect could be more remarkable in the future.

Moreover, the effect of congestion charging on matching performance were presented in section 4.1 where the matching rate and cost saving rate can reach $76.9 \%$ and $21.3 \%$, respectively. These results are in line with the general finding that "sticks" like parking charges seem to have a generally greater influence on traffic mode choice than "carrots" like preferential parking and subsidies (O'Fallon et al., 2004). 


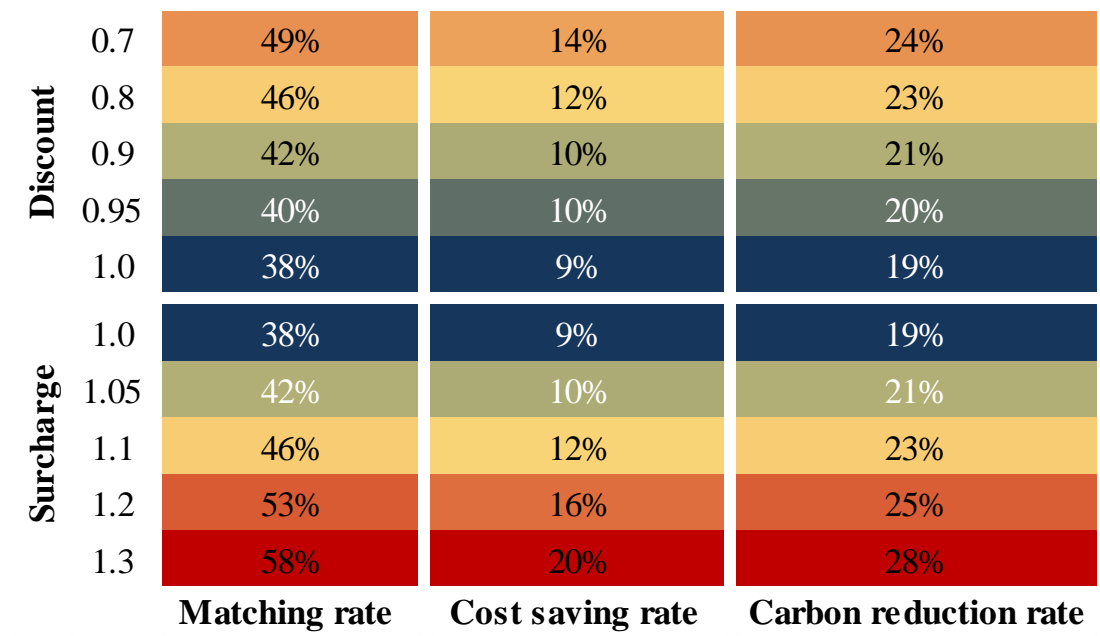

Fig. 15 The effect of parking fee discounts (the upper) and surcharges (the lower) on carpooling matching performance

\section{Discussion and Conclusions}

With the development of the urban economy and the growth of population, severe traffic congestion has been observed in many cities, which results in heavy economic losses due to the increase in travel time and energy consumption. By introducing high flexibility on trips and travel times, and leveraging the shareability of a journey (Santi et al., 2014), carpooling services could provide timely and convenient transportation using fewer cars and thus relieve the problems of urban roadways. Although both governments and employers promote carpooling as a commuting alternative, city-wide carpooling success stories are still in short supply. Private-car based carpooling may have greater potential in terms of carrying capacity and be more sustainable, and could create less regulatory challenges than ride-hailing based carpooling; hence private-car based carpooling may have a higher potential for scalable practical development. Focusing on the characteristics of private-car based carpooling and the motivations of potential carpoolers, we proposed a passenger-to-driver based carpooling matching model and formulated the matching process as a mixed-integer linear programming (MILP) problem. Then we investigated the sensitivity of this matching system based on actual data. Some major findings in this work are summarized as follows.

(1) Matching results show that $38.3 \%$ of trips within Outer London could successfully become carpooled trips, while $76.9 \%$ of car trips can be shared within central London because of the congestion charge. Moreover, carpooling can result in significant fuel savings with a carbon emissions reduction rate of up to $56 \%$ and a total saving of up to 11.8 metric tons $\mathrm{CO}_{2}$ per day.

(2) Various sharing patterns in carpooling trips bring about different matching results; a longer maximum additional walk time in the matching process can reduce generalized cost savings but increase total carbon emissions reductions. 
(3) Carpooling matching performance shows a sharp decline when the system participant rate drops from $40 \%$ to $20 \%$, therefore, it is necessary to obtain a moderate participant level in the start-up phase of a carpooling system. Participant flexibility, in terms of a willingness to be a driver or rider, to change schedule and accept detour, has a positive impact on the rate of successful matches, especially when participant numbers are low. Therefore, it is beneficial for providers to attract participants with higher flexibilities for role, detour and schedule.

(4) A lower detour time threshold and higher walking penalty factor can significantly damage the functioning of the carpooling system. Encouraging commuters to walk more can not only help form more carpooling trips but also reduce carbon emissions from each carpooling trip.

(5) With the impact of an additional sharing psychological penalty and detour penalty on the trip costs of riders, the optimal fee-sharing ratio for the trip matching rate and cost-based carpooling fairness is not the traditional half to half, but between $30 \%$ and $40 \%$. When the penalty factor rises, riders should pay less money since they have already paid a greater psychological cost or physical effort to carpool. Without eroding the matching performance much, we can set a moderate sharing psychological penalty factor to reduce this loss, but set a relatively high schedule deviation penalty factor to ensure time reliability of the carpooling service.

This study can be regarded as a starting point with respect to research on the private-car based carpooling matching model. However, there are some limitations of this study that should be discussed. First, it is still unclear what car commuters' attitudes are concerning the proposed carpooling mode in the real-world. Hence, we do not consider the various trip costs including sharing psychological penalty, detour penalty, and time deviation penalty, to be close to reality, but explore the impact of lower system participant numbers on matching performance. Considering some participants do not save much from carpooling and may quit this service, we exclude carpooling trips with cost savings of less than $10 \%$ of the total trip cost from the feasible trip set; this threshold limit only causes a $2 \%$ reduction in matching rate compared with the basic results presented in Section 4.1. Hence if these car commuters are not willing to share their trips because of low cost savings, the erosion of matching performance of this model is acceptable. Second, we neglect individual preferences and participants' sociodemographic characteristics like gender, age and employment status when setting the parameters and constraints in our matching model. Fortunately, earlier research tends to suggest that demographic factors do not strongly influence carpooling uptake (Canning et al., 2010; Vanoutrive et al., 2012). Third, we have not mapped the commuting trips data onto the local road network, this can create slight deviations when using zone-based travel time data and estimating walking time. In this carpooling system, it does not matter whether the method can identify the pick-up that is actually closest in terms of walking time, as long as the passengers do not need to walk for a significantly longer (or shorter) time to reach the allocated meeting point. We may get results with an acceptable accuracy level with less computational cost. Moreover, it 
may be difficult for both carpoolers to arrive at the meeting point at the same time, which may induce extra waiting time costs. We found that $85 \%$ of carpooling trips have a driving deviation time of less than 2 minutes during the drive to pick up their rider. Considering the high time reliability of walking trip stages, the impact of waiting time costs could be less significant. Lastly, the proposed matching model is more applicable to urban areas where the cost of car trips is expensive enough to motivate commuters to look pro-actively for alternatives to driving alone.

There is more work ahead in the future development of this study. First, we assume that only one passenger can be assigned to a carpooling driver. If drivers have sufficient time flexibility, they may be willing to provide rides to several riders on a trip, either one after the other or simultaneously for portions of the journey (Agatz et al, 2012). Second, we propose a static matching mode focusing on morning commuting trips without rolling planning horizons. However, the modeling framework in this paper can be easily extended to dynamic carpooling throughout 24 hours for various travel purposes; we can also consider the associated return trips in the carpooling system by matching trips in two directions or each direction separately. Third, we present the estimation method of sharing psychological cost in accordance with some theoretical assumptions and regional surveys, the parameters and effect of which need subsequent examination. It will also be interesting to investigate the impact of increasing acquaintance levels on matching performance by simulating repetitive matches over time or analyzing empirical data from real-world carpooling services in further studies. Another topic is to introduce cycling or public transport as detouring methods in longdistance carpooling trips (e.g. inter-city trips) and then compare these matching performances. Lastly, sometimes the platforms need to consider multiple objectives when matching passengers and drivers other than just for maximal operation profit, e.g. service quality (Lyu et al., 2019), and the agency-based platform may need to consider social welfare in the carpooling system. Hence it is necessary to integrate these focuses into the model objective and adaptively balance the trade-off between multiple objectives. In other words, this study builds a solid foundation for future research about developing a practicable and sustainable carpooling matching model.

\section{Acknowledgements}

This work is financially supported by National Natural Science Foundation of China (91746201, 71621001). Data retrieved from Uber Movement, (c) 2019 Uber Technologies, Inc., https://movement.uber.com. Many thanks to Transport for London and Uber Movement for providing the data in this study.

\section{Conflicts of Interest}

The authors declare that there is no conflict of interest in any aspect of the data collection, analysis, or the funding received regarding the publication of this paper. 


\section{References}

Abrantes, P. A., \& Wardman, M. R. (2011). Meta-analysis of UK values of travel time: An update. Transportation Research Part A: Policy and Practice, 45(1), 1-17.

Agatz, N.A.H., Erera, A.L., Savelsbergh, M.W.P., Wang, X., 2011. Dynamic ride-sharing: a simulation study in Metro Atlanta. Transportation Research Part B 45(9), 1450-1464.

Agatz, N., Erera, A., Savelsbergh, M., \& Wang, X. (2012). Optimization for dynamic ride-sharing: A review. European Journal of Operational Research, 223(2), 295-303.

Amey, A.M. 2011. Proposed methodology for estimating rideshare viability within an organization: Application to the MIT community. No. 11-2585.

Amirkiaee, S. Y., \& Evangelopoulos, N. (2018). Why do people rideshare? An experimental study. Transportation research part F: traffic psychology and behaviour, 55, 9-24.

Bachmann F, Hanimann A, Artho, Jürg, Jonas K, 2018. What drives people to carpool? Explaining carpooling intention from the perspectives of carpooling passengers and drivers [J]. Transportation Research Part F: Traffic Psychology and Behaviour. 59:260-268.

Buliung, R., Soltys, K., Bui, R., Habel, C., Lanyon, R., 2010. Catching a ride on the information super-highway: toward an understanding of internet-based carpool formation and use. Transportation 37 (6), 849-873.

Canning, P.E., Hughes, S.J., Hellawell, E.E., Gatersleben, B.C.M., Fairhead, C.J., 2010. Reasons for participating in formal employer-led carpool schemes as perceived by their users. Transportation Planning and Technology 33, 733-745.

Cetin, T., \& Deakin, E. (2017). Regulation of taxis and the rise of ridesharing. Transport Policy.

Chan, N. D., \& Shaheen, S. A. (2012). Ridesharing in North America: Past, present, and future. Transport Reviews, 32(1), 93-112.

Chaube, V., Kavanaugh, A. L., \& Perez-Quinones, M. A. (2010). Leveraging social networks to embed trust in rideshare programs. In 2010 43rd Hawaii International Conference on System Sciences (pp. 1-8). IEEE.

Correia, G., \& Viegas, J. M. (2011). Carpooling and carpool clubs: Clarifying concepts and assessing value enhancement possibilities through a Stated Preference web survey in Lisbon, Portugal. Transportation Research Part A: Policy and Practice, 45(2), 81-90.

Danish Ministry of Transport, (2013). Transportøkonomiske Enhedspriser. Transport ministeriet, Copenhagen.

Delhomme P, Gheorghiu A., 2016. Comparing French carpoolers and non-carpoolers: Which factors contribute the most to carpooling? [J]. Transportation Research Part D Transport \& Environment. 42:1-15.

Department for Transport. (2017a). Transport Statistics Great Britain: 2017 report summary: https://www.gov.uk/government/statistics/transport-statistics-great-britain-2017

Department for Transport. (2017b). National Travel Survey: 2017 factsheets: https://www.gov.uk/government/statistics/national-travel-survey-2017

Furuhata M, Dessouky M, Ordóñez F, Fernandoet al., 2013. Ridesharing: The state-of-the-art and future directions [J]. Transportation Research Part B Methodological. 57:28-46.

Galiza, R. J., Ferreira, L., \& Charles, P. (2011, January). Estimating the effects of older people in pedestrian flow: A micro-simulation approach. In Transport Research Board TRB 90th Annual Meeting (pp. 1-13).

García-Pelayo, R. (2005). Distribution of distance in the spheroid. Journal of Physics A: 
Mathematical and General, 38(16), 3475.

Gardner, B., \& Abraham, C. (2007). What drives car use? A grounded theory analysis of commuters' reasons for driving. Transportation Research Part F: Traffic Psychology and Behaviour, 10(3), 187200.

Greater London Authority. (2018). Mayors Transport Strategy 2018: https://www.london.gov.uk/sites/default/files/mayors-transport-strategy-2018.pdf

Hall, J. A. (2019). How many hours does it take to make a friend?. Journal of social and personal relationships, 36(4), 1278-1296.

Hong, Z., Chen, Y., Mahmassani, H. S., \& Xu, S. (2017). Commuter ride-sharing using topologybased vehicle trajectory clustering: Methodology, application and impact evaluation. Transportation Research Part C: Emerging Technologies, 85, 573-590.

Jacobson S H, King D M., 2009. Fuel saving and ridesharing in the US: Motivations, limitations, and opportunities [J]. Transportation Research Part D Transport \& Environment. 14(1):14-21.

Kamar, E., Horvitz, E., 2009. Collaboration and shared plans in the open world: studies of ridesharing. In: Proceedings of the Twenty-First International Joint Conference on Artificial Intelligence, AAAI, Palo Alto, California, USA. pp. 187-194.

Kwon, J., \& Varaiya, P. (2008). Effectiveness of California's high occupancy vehicle (HOV) system. Transportation Research Part C: Emerging Technologies, 16(1), 98-115.

Liu, H. X., \& Ma, W. (2009). A virtual vehicle probe model for time-dependent travel time estimation on signalized arterials. Transportation Research Part C: Emerging Technologies, 17(1), 11-26.

Liu, X., Yan, X., Liu, F., Wang, R., \& Leng, Y. (2019). A trip-specific model for fuel saving estimation and subsidy policy making of carpooling based on empirical data. Applied Energy, 240, 295-311.

Long, J., Tan, W., Szeto, W. Y., \& Li, Y. (2018). Ride-sharing with travel time uncertainty. Transportation Research Part B: Methodological, 118, 143-171.

Lyu, G., Cheung, W. C., Teo, C. P., \& Wang, H. (2019). Multi-Objective Online Ride-Matching. Available at SSRN 3356823.

Masoud, N., Jayakrishnan, R., 2017. A decomposition algorithm to solve the multi-hop Peer-to-Peer ride-matching problem. Transport. Res. B 99, 1-29.

Mcintosh J, Trubka R, Newman P, 2014. Can value capture work in a car dependent city? Willingness to pay for transit access in Perth, Western Australia [J]. Transportation Research Part A, 67(67):320-339.

Miritello, G., Moro, E., Lara, R., Martínez-López, R., Belchamber, J., Roberts, S. G., \& Dunbar, R. I. (2013). Time as a limited resource: Communication strategy in mobile phone networks. Social Networks, 35(1), 89-95.

Nie, Y. M., Wu, X., Dillenburg, J. F., \& Nelson, P. C. (2012). Reliable route guidance: A case study from Chicago. Transportation Research Part A: Policy and Practice, 46 (2), 403-419.

O'Fallon, C., Sullivan, C., Hensher, D.A., 2004. Constraints affecting mode choices by morning car commuters. Transport Policy 11, 17-29.

Polkowski, Z., \& Dysarz, J. (2016). An Analysis Of The Legal, Economic And Technical Aspects Of Carpooling Systems. Scientific Bulletin-Economic Sciences, 15(3), 76-88.

Polus, A. (1979). A study of travel time and reliability on arterial routes. Transportation, 8 (2), 141151. 
Roberts, S. G., \& Dunbar, R. I. (2011). The costs of family and friends: an 18-month longitudinal study of relationship maintenance and decay. Evolution and Human Behavior, 32(3), 186-197.

Rosenow, J., Guertler, P., Sorrell, S., \& Eyre, N. (2018). The remaining potential for energy savings in UK households. Energy policy, 121, 542-552.

Santi, P., Resta, G., Szell, M., Sobolevsky, S., Strogatz, S. H., \& Ratti, C. (2014). Quantifying the benefits of vehicle pooling with shareability networks. Proceedings of the National Academy of Sciences, 111(37), 13290-13294.

Shires, J. D., \& De Jong, G. C. (2009). An international meta-analysis of values of travel time savings. Evaluation and program planning, 32(4), 315-325.

Stiglic, M., Agatz, N., Savelsbergh, M., \& Gradisar, M. (2015). The benefits of meeting points in ride-sharing systems. Transportation Research Part B: Methodological, 82, 36-53.

Stiglic, M., Agatz, N., Savelsbergh, M., \& Gradisar, M. (2016). Making dynamic ride-sharing work: The impact of driver and rider flexibility. Transportation Research Part E: Logistics and Transportation Review, 91, 190-207.

Stiglic M, Agatz N, Savelsbergh M, 2018. Enhancing urban mobility: Integrating ride-sharing and public transit [J]. Computers \& Operations Research. 90:12-21.

Su, Q., \& Zhou, L. (2012). Parking management, financial subsidies to alternatives to drive alone and commute mode choices in Seattle. Regional science and urban economics, 42(1-2), 88-97.

Transport for London. (2018). Travel in London reports: Report 11, https://tfl.gov.uk/corporate/publications-and-reports/travel-in-london-reports\#on-this-page-0

US Environmental Protection Agency, 2016. Light-Duty Automotive Technology, Carbon Dioxide Emissions, and Fuel Economy Trends: 1975-2016.

United States Environmental Protection Agency and U.S. Department of Energy. (2017). Model Year 2018 Fuel Economy Guide-Electric vehicles \& Plug-in Hybrid Electric Vehicles: https://www.fueleconomy.gov/feg/pdfs/guides/FEG2018.pdf.

Vanoutrive T., Vijver E. V. D., Malderen L. V., Jourquin B., Thomas I., Verhetsel A.Vanoutrive T, Vijver E V D, Malderen L V, et al., Witlox F., 2012. What determines carpooling to workplaces in Belgium: location, organisation, or promotion? [J]. Journal of Transport Geography. 22(2):77-86.

Wang R., 2011. Shaping carpool policies under rapid motorization: the case of Chinese cities [J]. Transport Policy. 18(4):631-635.

Wang, X., Agatz, N., Erera, A., 2018. Stable matching for dynamic ride-sharing systems. Transport. Sci. 52 (4), 850-867.

Wang, Y., Winter, S., \& Ronald, N. (2017). How much is trust: The cost and benefit of ridesharing with friends. Computers, Environment and Urban Systems, 65, 103-112.

Wardman, M., Chintakayala, V. P. K., \& de Jong, G. (2016). Values of travel time in Europe: Review and meta-analysis. Transportation Research Part A: Policy and Practice, 94, 93-111.

Yongqi Dong, Shuofeng Wang, Li Li, Zuo Zhang, 2018. An empirical study on travel patterns of internet based ride-sharing [J]. Transportation Research Part C: Emerging Technologies. 86:1-22.

Zheng, F., \& Van Zuylen, H. (2013). Urban link travel time estimation based on sparse probe vehicle data. Transportation Research Part C: Emerging Technologies, 31, 145-157. 